\title{
A MODEL-BASED FAULT DETECTION AND DIAGNOSIS SCHEME FOR DISTRIBUTED PARAMETER SYSTEMS: A LEARNING SYSTEMS APPROACH *
}

\author{
Michael A. Demetriou ${ }^{1}$
}

\begin{abstract}
In this note, fault detection techniques based on finite dimensional results are extended and applied to a class of infinite dimensional dynamical systems. This special class of systems assumes linear plant dynamics having an abrupt additive perturbation as the fault. This fault is assumed to be linear in the (unknown) constant (and possibly functional) parameters. An observer-based model estimate is proposed which serves to monitor the system's dynamics for unanticipated failures, and its well posedness is summarized. Using a Lyapunov synthesis approach extended and applied to infinite dimensional systems, a stable adaptive fault diagnosis (fault parameter learning) scheme is developed. The resulting parameter adaptation rule is able to "sense" the instance of the fault occurrence. In addition, it identifies the fault parameters using the additional assumption of persistence of excitation. Extension of the adaptive monitoring scheme to incipient faults (time varying faults) is summarized. Simulations studies are used to illustrate the applicability of the theoretical results.
\end{abstract}

Mathematics Subject Classification. 93C20, 35B37, 93D21, 93D05.

Received April 11, 2000. Revised April 2, 2001.

\section{INTRODUCTION}

The motivation of this work has come from recent developments in the use of neural networks and adaptive estimation techniques for on-line failure detection and diagnosis of finite dimensional dynamical systems using a model-based scheme [51,54]. Extensions of these model-based schemes to infinite dimensional systems have not received considerable attention as in the finite dimensional case. Even though in the last two decades there has been an enormous development in the theory of control and estimation of infinite dimensional systems (both theoretical and computational), less attention has been paid to the adaptive estimation and control of distributed parameter systems. For adaptive control and estimation of infinite dimensional systems the reader is referred to $[8,13,15,40-42,61,63]$, to name a few, for various approaches where either a finite dimensional model of a plant was assumed and an adaptive controller was based on the approximated model, or when the infinite dimensional system was decomposed into a stabilizable finite dimensional subsystem to be controlled and an infinite dimensional stable system. For control and estimation of infinite dimensional systems the reader is

Keywords and phrases: Fault detection, distributed parameter systems.

* Research supported in part by the Air Force Office of Scientific Research under grant AFOSR F49620-93-1-0198 while the author was with the Center for Research for Scientific Computation, Raleigh, NC and in part by Faculty Development Grant at Worcester Polytechnic Institute.

1 Department of Mechanical Engineering, Worcester Polytechnic Institute, Worcester, MA 01609, U.S.A.;

e-mail: mdemetri@wpi.edu 
referred to the books $[4,10,11,14,19,39,44]$ for an in-depth exposition. One reason for this disparity (in minimal representation of literature work on adaptive control of infinite dimensional systems) was the enormous size of the systems involved (even their finite dimensional approximations) and their inherent complexities/nonlinearities introduced due to adaptation rules, which make their on-line implementation currently not feasible (partially feasible for special cases for 1-D and 2-D problems) in real-time.

With the advent of computing powers, computationally intensive algorithms for control and estimation of infinite dimensional systems are now becoming applicable, and with the careful and efficient choice of implementation algorithms, real-time implementable. It is due to this fact that we undertake such an ambitious project and extend the finite dimensional treatments of on-line fault detection schemes to a class of infinite dimensional systems. Even though the proposed scheme requires the full state of the infinite dimensional systems, the goal is to introduce the abstract mathematical framework necessary for the synthesis and convergence analysis of fault detection schemes. This would serve as a departure point for the more realistic case of partial observation (pointwise/boundary observations) of the state of a system whose dynamics are governed by equations evolving in abstract spaces.

In this paper an abstract framework for the on-line fault detection and diagnosis for a class of infinite dimensional dynamical systems (plants) is developed. The fault is modeled as an additive perturbation of the dynamics that is expressed as a parametrized operator evaluated at an unknown parameter. The fault (i.e. the additive perturbation) is assumed to commence at an unknown time instance. The nature of the additive perturbation in the dynamics is assumed to be known, but the parameter at which is evaluated is unknown and it is therefore desired to be identified. The state estimator, or detection observer, takes the form of an infinite dimensional linear evolution system with time varying coefficients. This state estimator uses as its inputs (i) the state of the plant (plant output) and (ii) the plant's adjustable parameters estimates (adaptive estimates). Using an argument based on Lyapunov redesign method [37,43], which essentially forces the time derivative of a Lyapunov functional to be non positive, the update laws (adaptation rules) for parameter adjustment are derived. The right choice of the online parameter laws guarantees the convergence of the state error to zero with no additional conditions imposed either on the state of the plant or the input to the system. By imposing additional conditions on the state of the plant, and implicitly on the input signal, parameter convergence can be established, and hence failure isolation, [35].

The combined state and parameter estimator purpose is twofold: (i) to serve as a monitor of the system dynamics and detect the time instance the failure occurs, and (ii) to diagnose the nature of the failure which in this case is assumed to be either a perturbation of the nominal dynamics or another operator whose structure is known but the parameter at which it is evaluated is unknown. Specifically, it is assumed that the failures are additive and are linear with respect to the parameters. No failures in the input term (actuator failure), commonly denoted by $B u(t)$ in the literature, are considered at this stage as this would be more relevant in the context of actuator failure and its consequent plant accommodation.

The approach here represents an infinite dimensional analogue of an automated fault detection scheme developed for finite dimensional systems in [51,54] and more recently in [30]. The design of the diagnostic observers falls under the category of model-based analytical redundancy approach. The survey papers by Frank [33], Gertler [34], Isermann [38] and Patton [49] provide detailed overviews of the various model-base fault detection algorithms. For an in-depth exposure the reader is directed to the books $[12,35,46]$. The convergence of the state error is obtained using a Lyapunov estimate in a fashion similar to the finite dimensional case. Due to the linearity of the parameters with respect to the additive failures, parameter convergence can be guaranteed using the notion of persistence of excitation.

An outline of the remainder of the paper is as follows. In Section 2, the problem is formulated in an abstract setting and the mathematical preliminaries are provided. The detection observer (estimated model) and the on-line fault (parameter) estimator are defined in a variational form. Convergence of the proposed adaptive monitoring scheme is investigated in Section 3 and extensions to the case of incipient failures (i.e. slowly developing) are presented in Section 4. Examples and results of numerical simulations are presented in 
Section 5. Conclusions with directions for future research on fault detection and accommodation of systems governed by partial differential equations are presented in Section 6 .

In general all notation is standard. For $X$ and $Y$ Banach spaces, $\mathcal{L}(X, Y)$ denotes the space of bounded linear operators from $X$ into $Y$. Also, for $X$ a linear space and $Y$ a space of linear functionals on $X,\langle\varphi, x\rangle_{X, Y}$ denotes the action of the linear functional $\varphi \in Y$ on the element $x \in X$.

\section{Problem formulation}

In this section a procedure for designing a fault detection scheme for a class of infinite dimensional systems is outlined. Specifically, we will be concerned with the following class of dynamical systems

$$
\dot{x}(t)+A x(t)+\beta\left(t-t^{*}\right) D(\theta) x(t)=B u(t), \quad x(0)=x_{0} \in H,
$$

where $H$ is an infinite dimensional space, $x$ denotes the state, and $A, D, B$ denote the system operator, the failure operator and the input operator, respectively. In this case, the failure is assumed to be abrupt [51], and specifically the function $\beta\left(t-t^{*}\right)$ that represents the time profile of the failure is assumed to be a step function that is given by

$$
\beta\left(t-t^{*}\right)= \begin{cases}1 & \text { for } t \geq t^{*} \\ 0 & \text { for } t<t^{*}\end{cases}
$$

The nominal system dynamics given in (1) via the term $A x(t)$, i.e. the system

$$
\dot{x}(t)+A x(t)=B u(t), \quad x(0)=x_{0} \in H,
$$

are assumed to be known. The $\theta$-parameterized operator $D(\theta)$ models the unanticipated failure and it is assumed that the structure of the failure is known, i.e. for a given parameter $\theta$ the operator $D(\cdot)$ is known, but the parameter $\theta$ is unknown. Below, we will provide the mathematical preliminaries required for the analysis and well-posedness of the plant and the derivation of the on-line estimated model of (1). This estimated model, or detection observer, will use as its inputs the output of the plant $x(t)$ and the adjustable (on-line) estimates $\hat{\theta}(t)$ of the (unknown) parameter $\theta$. Following the definition from finite dimensional treatments on failure detection, the estimated model has what is termed as error filtering model which is similar to the linear observer design [47] and to the estimation schemes for finite dimensional systems in $[52,53,56]$.

It will be shown in the following section that the proposed estimated model can detect the time of failure $t^{*}$ and, by imposing the additional condition of persistence of excitation, the parameter $\theta$ in the additive term $D(\theta) x(t)$ will be identified asymptotically with time. This will also be demonstrated via some numerical simulations in Section 5, where the time $t^{*}$ of the failure will be "sensed" by this detection observer and the parameter error $\widehat{\theta}(t)-\theta$ will asymptotically converge to zero in an appropriate norm.

\subsection{Plant in variational form}

We will consider the above equation (1) in weak or variational form. Towards this end, let $H$ be a Hilbert space with inner product $\langle\cdot, \cdot\rangle$ and corresponding norm $|\cdot|$. We also let $V$ be a reflexive Banach space with norm denoted by $\|\cdot\|$, and assume that $V$ is embedded densely and continuously in $H$. We let $V^{*}$ denote the conjugate dual of $V$ (i.e. the space of continuous conjugate linear functionals on $V$ ) with norm denoted by $\|\cdot\|_{*}$ (i.e. the usual uniform operator norm). It then follows that $V \hookrightarrow H \hookrightarrow V^{*}$ with both embeddings dense and continuous. We then have that

$$
|\varphi| \leq K\|\varphi\|, \quad \varphi \in V,
$$


for some positive (embedding) constant $K[45,59,60,64]$. The notation $\langle\cdot, \cdot\rangle$ will also be used to denote the duality pairing between $V^{*}$ and $V$ induced by the continuous and dense embeddings in (4).

The parameter space is denoted by $Q$ and it is assumed to be a Hilbert space with inner product $\langle\cdot, \cdot\rangle_{Q}$ and norm $|\cdot|_{Q}$. The dual $Q^{*}$ of $Q$ is identified by $Q^{*}=Q$. For each $\theta \in Q$, let $D(\theta): V \rightarrow V^{*}$ be an operator satisfying the following assumptions

(D1) ( $Q$-Linearity) The map $\theta \rightarrow D(\theta) \varphi$ is linear from $Q$ into $V^{*}$ for each $\varphi \in V$.

(D2) $\left(V-V^{*}\right.$-Boundedness) There exists a scalar $\alpha_{d}>0$ such that for each $\theta \in Q$

$$
|\langle D(\theta) \varphi, \psi\rangle| \leq \alpha_{d}|\theta|_{Q}\|\varphi\|\|\psi\|, \quad \varphi, \psi \in V .
$$

Remark 2.1. The fact that the parameter space $Q$ is chosen to be a Hilbert space (as opposed to a Euclidean space), enables the proposed scheme to identify functional parameters in the failure term $D(\theta) x(t)$.

Continuing, we consider the rather standard assumptions on the nominal plant operator $A$ that are required for the existence and uniqueness of solutions to the nominal system (3).

(A1) $\left(V-V^{*}\right.$-Boundedness) There exists a scalar $\alpha_{a}>0$ such that

$$
|\langle A \varphi, \psi\rangle| \leq \alpha_{a}\|\varphi\|\|\psi\|, \quad \varphi, \psi \in V .
$$

(A2) $\left(V-H\right.$-Coercivity) There exists a $\lambda_{a} \in \mathbb{R}$ and a scalar $\beta_{a}>0$ such that

$$
\mathcal{R} e\langle A \varphi, \varphi\rangle+\lambda_{a}|\varphi|^{2} \geq \beta_{a}\|\varphi\|^{2}, \quad \varphi \in V .
$$

In addition, we consider the operator $L: V \rightarrow V^{*}$, appearing in the detection/diagnostic observer model below, which satisfies the following assumptions.

(L1) $\left(V-V^{*}\right.$-Boundedness) There exists a scalar $\alpha_{l}>0$ such that

$$
|\langle L \varphi, \psi\rangle| \leq \alpha_{l}\|\varphi\|\|\psi\|, \quad \varphi, \psi \in V .
$$

(L2) ( $V$-Coercivity) There exists a scalar $\beta_{l}>0$ such that

$$
\mathcal{R} e\langle L \varphi, \varphi\rangle \geq \beta_{l}\|\varphi\|^{2}, \quad \varphi \in V .
$$

Remark 2.2. The strict $V$-coercivity imposed on the design operator $L$ is to affect and guarantee the asymptotic convergence of the state estimation error. This, in a way, is parallel to the design matrix $A_{m}$ (error filtering model with series-parallel configuration) often appearing in the finite dimensional treatment of adaptive estimation and control, see for example $[37,43,48]$.

In addition, for $\varphi \in V$ we define the linear operator $G(\varphi): V \rightarrow Q$ by

$$
\langle G(\varphi) \psi, \theta\rangle_{Q}=\langle D(\theta) \varphi, \psi\rangle, \quad \psi \in V, \quad \theta \in Q .
$$

Using Assumption (D2), it is clear that for $\varphi \in V$, we have $G(\varphi) \in \mathcal{L}(V, Q)$ with

$$
\|G(\varphi)\|_{\mathcal{L}(V, Q)} \leq a_{d}\|\varphi\| .
$$

Using equation (5) we define, for $\varphi \in V$, the Banach space adjoint of the operator $G(\varphi)$, denoted here by $G^{*}(\varphi) \in \mathcal{L}\left(Q, V^{*}\right)$, as

$$
\left\langle G^{*}(\varphi) \theta, \psi\right\rangle_{V^{*}, V}=\langle G(\varphi) \psi, \theta\rangle_{Q}, \quad \psi \in V, \quad \theta \in Q .
$$


Let the initial data $x(0)=x_{0} \in H$ and the input, or control, term $B u(t) \in L_{2}\left(0, T ; V^{*}\right)$, and consider the initial value problem

$$
\begin{gathered}
\dot{x}(t)+A x(t)+\beta\left(t-t^{*}\right) D(\theta) x(t)=B u(t), \quad \text { a.e. } t>0, \\
x(0)=x_{0} .
\end{gathered}
$$

We establish the well-posedness of the above system via the existence of a weak solution. First, we show the well posedness of the (nominal) system for $t<t^{*}$, i.e. prior to the failure, and then establish the well posedness of the system with the failure incorporated into its dynamics. Specifically, we first consider the system

$$
\text { (I) }\left\{\begin{array}{l}
\dot{x}(t)+A x(t)=B u(t), \\
x(0)=x_{0},
\end{array}\right.
$$

and then (using (2)) the system

$$
\left\{\begin{array}{l}
\dot{x}(t)+A x(t)+D(\theta) x(t)=B u(t), \quad t \geq t^{*} \\
x\left(t^{*}\right)=x_{*} .
\end{array}\right.
$$

By a weak solution to the initial value problem (9) we mean a function $x \in L_{2}(0, T ; V)$ with $\dot{x} \in L_{2}\left(0, T ; V^{*}\right)$ for all $0<T<t^{*}$ that satisfies (9). Similarly, by a weak solution to (10) we mean a function $x \in L_{2}\left(t^{*}, T\right.$; $V$ ) with $\dot{x} \in L_{2}\left(t^{*}, T ; V^{*}\right)$ for all $T \geq t^{*}$ that satisfies (10). Sufficient conditions that guarantee the existence of a unique solution are presented in $[11,44,45,50,59,60,64]$. Specifically, the operator $A$ being coercive and bounded with $B u(t) \in L_{2}\left(0, T ; V^{*}\right)$, are sufficient conditions to guarantee the existence of a unique solution to (9). Similarly, if the operator $[A+D(\theta)]$ is coercive and bounded for all $\theta \in Q$ with $B u(t) \in L_{2}\left(0, T ; V^{*}\right)$, are sufficient conditions for the existence of solutions to (10). The former condition can be satisfied if

$$
\begin{aligned}
\mathcal{R} e\langle(A+D(\theta)) \varphi, \varphi\rangle+\lambda_{a}|\varphi|^{2} & \geq \mathcal{R} e\langle A \varphi, \varphi\rangle-|\langle D(\theta) \varphi, \varphi\rangle|+\lambda_{a}|\varphi|^{2} \\
& \geq \beta_{a}\|\varphi\|^{2}-a_{d}|\theta|_{Q}\|\varphi\|^{2} \geq\left(\beta_{a}-a_{d}|\theta|_{Q}\right)\|\varphi\|^{2}
\end{aligned}
$$

which would require $\beta_{a}>a_{d}|\theta|_{Q}$.

\subsection{Estimated model and a learning scheme}

Before we present the state estimator, we give the definition of a bounded plant, which in a way is a uniform boundedness condition on $\|x(t)\|$.

Definition 2.3 (Bounded plant). A bounded plant is a pair $(\theta, x)$ with $x$ a solution to $(7,8)$ for which there exists a constant $\gamma=\gamma(x)$ such that

$$
\left|\langle G(x(t)) \varphi, \theta\rangle_{Q}\right| \leq \gamma(x(t))|\theta|_{Q}\|\varphi\|, \quad t>t^{*}, \quad \theta \in Q, \varphi \in V
$$

It should be noted that if the pair $(\theta, x)$ is a bounded plant, then we have that $G(x(\cdot)) \in L_{2}\left(t^{*}, T ; \mathcal{L}(V, Q)\right)$ for all $T>t^{*}$. It also follows from equation (6) that if $(\theta, x)$ is such that $\|x(t)\| \leq \gamma<\infty$, a.e. $t>t^{*}$, for some $\gamma>0$, then $(\theta, x)$ is indeed a bounded plant. In [13], it was shown that it is possible to provide sufficient conditions for the uniform boundedness of $\|x(t)\|$ for $t>t^{*}$.

We can now propose the estimated model of $(7,8)$ along with the adaptive law for the adjustment of the parameter estimates (i.e. adaptive detection/diagnostic observer). 
They take the form of an initial value problem and are given by

$$
\begin{gathered}
\dot{\hat{x}}(t)+L \widehat{x}(t)+G^{*}(x(t)) \widehat{\theta}(t)=B u(t)-A x(t)+L x(t), \\
\dot{\hat{\theta}}(t)-G(x(t)) \widehat{x}(t)=-G(x(t)) x(t), \quad \text { a.e. } \quad t>0, \\
\widehat{x}(0)=x(0), \quad \widehat{\theta}(0)=0 .
\end{gathered}
$$

To establish the well posedness of the (state and parameter) estimator (11-13), we follow a procedure similar to the one taken for the adaptive parameter estimation of distributed parameter systems in [20]. We let $X=H \times Q$ and $Y=V \times Q$. When $X$ and $Y$ are endowed with the usual product norm topologies, $X$ becomes a Hilbert space and $Y$ a reflexive Banach space. We then have the dense and continuous embeddings $Y \hookrightarrow X \hookrightarrow Y^{*}$. We define the operator $\mathcal{A}(t): Y \rightarrow Y^{*}$ by

$$
\mathcal{A}(t)=\left[\begin{array}{cc}
L & G^{*}(x(t)) \\
-G(x(t)) & 0
\end{array}\right]
$$

and the input (forcing) term $\mathcal{F}(t) \in Y^{*}$ by

$$
\mathcal{F}(t)=\left[\begin{array}{c}
B u(t)-A x(t)+L x(t) \\
-G(x(t)) x(t)
\end{array}\right]
$$

for almost every $t>0$.

The fact that $(\theta, x)$ is a bounded plant (Def. 2.3) and $B u(t) \in L_{2}\left(0, T ; V^{*}\right)$ implies that $\mathcal{F} \in L_{2}\left(0, T ; Y^{*}\right)$ for all $T>0$. Assumptions $(L 1)$ and $(L 2)$ together with $(\theta, x)$ being a bounded plant imply that $\mathcal{A}(t) \in \mathcal{L}\left(Y, Y^{*}\right)$, $t>0$ and that for $t>0$

$$
\mathcal{R} e\langle\mathcal{A}(t) \varphi, \varphi\rangle_{Y^{*}, Y}+\rho|\varphi|_{X}^{2} \geq \sigma\|\varphi\|_{Y}^{2}, \quad \varphi \in Y
$$

where $|\cdot|_{X}$ and $\|\cdot\|_{Y}$ denote respectively, the norms on $X$ and $Y$, and $\rho, \sigma>0$. It follows (see, for example [44, $45,59,60,64])$ that the initial value problem

$$
\begin{aligned}
& \dot{\xi}(t)+\mathcal{A}(t) \xi(t)=\mathcal{F}(t), \quad \text { a.e. } t>0, \\
& \xi(0) \in X,
\end{aligned}
$$

admits a unique solution $\xi \in L_{2}(0, T ; Y)$ with $\dot{\xi} \in L_{2}\left(0, T ; Y^{*}\right)$, all $T>0$. Consequently, the estimator (11-13) admits a unique solution $(\widehat{\theta}, \widehat{x}) \in L_{2}(0, T ; Q) \times L_{2}(0, T ; V)$ with $(\dot{\hat{\theta}}, \dot{\hat{x}}) \in L_{2}(0, T ; Q) \times L_{2}\left(0, T ; V^{*}\right)$, all $T>0$. Moreover, for each $T>0, \widehat{\theta}$ and $\widehat{x}$ agree almost everywhere with functions in $C([0, T] ; Q)$ and $C([0, T] ; H)$, respectively.

We denote the output estimation error or state error, by $e(t)=\widehat{x}(t)-x(t)$ and the parameter estimation error or parameter error, by $r(t)=\widehat{\theta}(t)-\theta$. Using the linearity assumption $(D 1)$ and the fact that for $t<t^{*}$ the parameter $\theta \equiv 0$, we have

$$
\begin{aligned}
\beta\left(t-t^{*}\right) D(\theta) x(t) & =0 \cdot D(\theta) x(t), \quad t<t^{*} \\
& =D(0) x(t)
\end{aligned}
$$

and that for $t \geq t^{*}$

$$
\begin{aligned}
\beta\left(t-t^{*}\right) D(\theta) x(t) & =1 \cdot D(\theta) x(t), \quad t \geq t^{*} \\
& =D(\theta) x(t)
\end{aligned}
$$


we can then write the error equations as

$$
\begin{gathered}
\dot{e}(t)+L e(t)+G^{*}(x(t)) r(t)=0, \quad \text { a.e. } t>0, \\
\dot{r}(t)-G(x(t)) e(t)=0, \quad \text { a.e. } t>0,
\end{gathered}
$$

where for $t<t^{*}$ the parameter error is given by $r(t)=\widehat{\theta}(t)-0$ and for $t \geq t^{*}$ it is given by $r(t)=\widehat{\theta}(t)-\theta$. The initial conditions are given by

$$
e(0)=\widehat{x}(0)-x(0)=x(0)-x(0)=0, \quad r(0)=\widehat{\theta}(0)-0=0 .
$$

Equivalently, the error equations $(16,17)$ can be written as

$$
\frac{\mathrm{d}}{\mathrm{d} t}\left[\begin{array}{l}
e(t) \\
r(t)
\end{array}\right]+\mathcal{A}(t)\left[\begin{array}{l}
e(t) \\
r(t)
\end{array}\right]=0, \quad \text { a.e. } t>0
$$

with the operator $\mathcal{A}(t)$ given by (14).

The choice $\hat{\theta}(0)=0$ will be explained in the next section where it will be shown that for $t<t^{*}$ the parameter estimator $\widehat{\theta}$ will estimate the zero parameter (i.e. $\theta(t)=0)$ and at $t \geq t^{*}$ will adaptively estimate the nonzero parameter $\theta$. In addition, the choice $\widehat{x}(0)=x(0)$ will be shown to guarantee that for $t<t^{*}, e(t) \equiv 0$ and for $t \geq t^{*},|e(t)| \geq 0$. The latter is a means of sensing the failure in the system, i.e. when $e(t)$ becomes nonzero it means that the system dynamics changed from $A x(t)$ to $[A+D(\theta)] x(t)$. With no additional assumptions, it will be shown that the state error $e(t)$ converges to zero asymptotically after the failure occurs. Additionally, the parameter estimate will be shown to estimate the zero parameter (i.e. $\widehat{\theta}$ remain at zero) for $t<t^{*}$ and after the failure occurs, it will attempt to estimate the parameter $\theta$. In order to guarantee that the parameter estimator will asymptotically estimate the parameter $\theta$ after the failure, we must impose the additional assumption of persistence of excitation, see [43,48].

\section{Convergence of the Learning scheme}

In this section, we make the standing assumption that the pair $(\theta, x)$ is a bounded plant (Def. 2.3). We use a Lyapunov-like argument to show convergence of the state error $e(t)$ to zero. Toward this end, we define the function $E:[0, \infty) \rightarrow \mathbb{R}^{+}$by

$$
E(t)=\frac{1}{2}\left\{|e(t)|^{2}+|r(t)|_{Q}^{2}\right\}, \quad t \geq 0
$$

As a first result we get a bound on this energy function $E(t)$.

Lemma 3.1. For all $t<t^{*}$ we have

$$
E(t)+\beta_{l} \int_{0}^{t}\|e(\tau)\|^{2} \mathrm{~d} \tau \leq E(0)
$$

and for $t>t^{*}$ we have

$$
E(t)+\beta_{l} \int_{t^{*}}^{t}\|e(\tau)\|^{2} \mathrm{~d} \tau \leq E\left(t^{*}\right) .
$$

Proof. Using (16-18) and Assumption (L2) we have that for $t<t^{*}$

$$
\frac{\mathrm{d}}{\mathrm{d} t} E(t)=\left\langle\frac{\mathrm{d}}{\mathrm{d} t} e(t), e(t)\right\rangle+\left\langle\frac{\mathrm{d}}{\mathrm{d} t} r(t), r(t)\right\rangle=-\langle L e(t), e(t)\rangle \leq-\beta_{l}\|e(t)\|^{2} .
$$


When equation (23) is integrated from 0 to some $t<t^{*}$ we obtain the desired result (21). Similarly, when we integrate from $t^{*}$ to some $t \gg t^{*}$ we get $(22)$.

The above lemma is used to show that the state error $e(t)$ either remains at zero for $t<t^{*}$ or it assumes a nonzero value at $t^{*}$ (jump) and converges asymptotically to zero for some $t \gg t^{*}$. This is stated as a theorem below.

Theorem 3.2. The error equations (16-18) that result by combining the plant $(7,8)$ with the detection/diagnostic observer (11-13), satisfy:

(i) for $t<t^{*}$ we have $E(t) \equiv e(t) \equiv r(t) \equiv 0$;

(ii) for $t>t^{*}$ the function $E(t)$ is nonincreasing and $\lim _{t \rightarrow \infty}|e(t)|=0$.

Proof. (Case (i), $t<t^{*}$.) Using the fact that at $t=0$ the initial conditions $e(0)=\widehat{x}(0)-x(0)=0$ and $r(0)=\widehat{\theta}(0)-0=0$, and the result of Lemma 3.1, we have that

$$
E(t)+\beta_{l} \int_{0}^{t}\|e(\tau)\|^{2} \mathrm{~d} \tau \leq E(0) \equiv 0, \quad t<t^{*}
$$

which implies that $E(t) \equiv 0$ for all $t<t^{*}$.

(Case (ii), $t>t^{*}$.) Using equation (22)

$$
E(t)+\beta_{l} \int_{t^{*}}^{t}\|e(\tau)\|^{2} \mathrm{~d} \tau \leq E\left(t^{*}\right), \quad t>t^{*}
$$

we have that $E$ (with $E\left(t^{*}\right) \neq 0$ ) is nonincreasing. The convergence of $|e(t)|$ to zero follows from the same arguments used in [20] for the adaptive parameter estimation of distributed parameter systems. It is essentially based on Barbălat's lemma [55] often used in the adaptive estimation and control of finite dimensional systems, $[43,48,55]$.

Remark 3.3. It can be observed from Theorem 3.2 that the estimator will sense the time $t^{*}$ of failure, since the state error is identical to zero for all time $t$ up to failure time $t^{*}$. The state error becomes nonzero after $t^{*}$ and converges to zero afterwards. When the state error attains a nonzero value it indicates that the failure occurred and hence the time $t^{*}$ can be detected by monitoring the state error $e(t)$. Another way to detect the failure is by monitoring the parameter estimate $\widehat{\theta}(t)$, as it too remains at zero for $t<t^{*}$ and becomes nonzero thereafter.

Remark 3.4. Another way of showing asymptotic convergence of the state error to zero, is to use a method employed in a lemma by Curtain and Oostveen in [18]. Basically, it uses the fact that the operator $L$ generates an exponentially stable $C_{0}$ semigroup and that $x$ is square integrable along with the boundendness of $r$ in equation (16).

Remark 3.5. In the above design, it was assumed that the initial condition $x(0)$ was known. If this is not known, one can actually built a monitoring observer of the healthy system in the infinitely remote past and assume that the state error $e(t)$ is relaxed at time $t_{0},[36]$. Alternatively, if an upper bound on the norm of $e\left(t_{0}\right)$ is known, then a dead-zone adaptive law can be augmented in the design to ensure that false alarms due to nonzero $e\left(t_{0}\right)$ are avoided, see $[30,51,54]$ for the finite dimensional treatment and [32] for the infinite dimensional case.

The convergence of $\widehat{\theta}(t)$ to the actual parameter $\theta$ is established by imposing the additional assumption of persistence of excitation, $[43,48]$. Below, we provide the equivalent definition of this persistence of excitation condition as it extends to infinite dimensional systems, [20]. 
Definition 3.6 (Persistence of Excitation). A bounded plant $(\theta, x)$ is said to be persistently excited, if there exists $T_{0}, \delta_{0}, \epsilon_{0}>0$ such that for each $p \in Q$ with unit norm (i.e. $|p|_{Q}=1$ ) and each $t>0$ sufficiently large (in this case $\left.t \gg t^{*}\right)$, there exists a $\tilde{t} \in\left[t, t+T_{0}\right]$ such that

$$
\left\|\int_{\tilde{t}}^{\widetilde{t}+\delta_{0}} G^{*}(x(\tau)) p \mathrm{~d} \tau\right\|_{*} \geq \epsilon_{0},
$$

where $G^{*}(x(t)) \in \mathcal{L}\left(Q, V^{*}\right)$ is the Banach space adjoint of the operator defined in (5).

Remark 3.7. Using equation (5) we can write the above persistence of excitation condition (24) as

$$
\begin{aligned}
\left\|\int_{\tilde{t}}^{\tilde{t}+\delta_{0}} G^{*}(x(\tau)) p \mathrm{~d} \tau\right\|_{*} & =\sup _{\|\phi\| \leq 1}\left|\int_{\tilde{t}}^{\widetilde{t}+\delta_{0}}\left\langle G^{*}(x(\tau)) p, \phi\right\rangle_{V^{*}, V} \mathrm{~d} \tau\right|=\sup _{\|\phi\| \leq 1}\left|\int_{\tilde{t}}^{\tilde{t}+\delta_{0}}\langle G(x(\tau)) \phi, p\rangle_{Q} \mathrm{~d} \tau\right| \\
& =\sup _{\|\phi\| \leq 1}\left|\int_{\tilde{t}}^{\tilde{t}+\delta_{0}}\langle D(p) x(\tau), \phi\rangle \mathrm{d} \tau\right| \geq \epsilon_{0}, \quad \phi \in V .
\end{aligned}
$$

Theorem 3.8. If the plant $(q, x)$ is persistently excited then

$$
\lim _{t \rightarrow \infty}|r(t)|_{Q}=\lim _{t \rightarrow \infty}|\widehat{\theta}(t)-\theta|_{Q}=0
$$

Proof. The proof is identical to the one used for the adaptive parameter identification of infinite dimensional dynamical systems in [13] and it is therefore omitted.

Remark 3.9. In the case that the operator $A$ satisfies a (stronger) coercivity assumption like Assumption ( $L 2)$, i.e. $V$-coercive, then we can set $L \equiv A$ in (11) to get a simplified estimator

$$
\dot{\widehat{x}}(t)+A \widehat{x}(t)+G^{*}(x(t)) \widehat{\theta}(t)=B u(t)
$$

This of course would affect the convergence properties of the estimator since the error equation (16) is now given by

$$
\dot{e}(t)+A e(t)+G^{*}(x(t)) r(t)=0, \quad \text { a.e. } t>0,
$$

and due to assumption $(A 2)$, the constant $\beta_{a}$ can not be chosen in a way to affect the convergence of $|e(t)|$ to zero in a desired way. An in-depth study of the effects of the choice of the constant $\beta_{l}$ on the speed of convergence of $|e(t)|$ to zero and its relation to the speed of convergence of $|r(t)|$ to zero was presented in [31] for the adaptive parameter estimation of parabolic and hyperbolic distributed parameter systems and in [9] in the context of hyperbolic distributed parameter systems with time varying parameters. In summary, a large value of $\beta_{l}$ in assumption $(L 2)$ would introduce a form of a high gain with oscillatory behavior and faster convergence of the state error to zero whereas a small value of $\beta_{l}$ would affect the convergence of the parameter error to zero.

Remark 3.10. It is interesting to note that persistence of excitation implies identifiability of the parameter $\theta$, see [10]. For if the plant is persistently excited and the parameter $\theta$ is not identifiable, then there would exist $\theta_{1}, \theta_{2} \in Q$ such that $x(t)$ is a solution to the initial value problem (10) (with $t_{0}=t^{*}$ and initial condition $x\left(t_{0}\right)=x\left(t^{*}\right)$ ) with either $\theta=\theta_{1}$ or $\theta=\theta_{2}$. Subtracting the two equations (that correspond to $\theta_{1}$ and $\theta_{2}$ ), we have that $\left\langle D\left(\theta_{1}-\theta_{2}\right) x(t), \varphi\right\rangle=0$, a.e. $t \geq t^{*}, \varphi \in V$, or using (5), that $G^{*}(x(t))\left(\theta_{1}-\theta_{2}\right)=0$, a.e. $t \geq t^{*}$. This of course contradicts Definition 3.6, and therefore identifiability is concluded. 


\section{EXtensions to TIME-VARYING PARAMETERS}

In this section we briefly describe an extension of the above learning scheme. We will consider the case where the nature of failure is incipient (slowly developing), see [51], and in this case the function $\beta\left(t-t^{*}\right)$ (time profile of the failure) is assumed to be of the following form

$$
\beta\left(t-t^{*}\right)=\left\{\begin{array}{ll}
1+\kappa \mathrm{e}^{-\alpha\left(t-t^{*}\right)} & \text { for } t \geq t^{*} \\
0 & \text { for } t<t^{*}
\end{array}, \quad \kappa, \alpha>0 .\right.
$$

This in a way restricts the type of temporal variations of the parameter $\theta$. This time profile corresponds to an exponentially distributed random variable which models the failure occurrence, [17]. The parameter is assumed to exponentially converge to some steady state value $\theta_{s s}$. The finite dimensional approach to time varying plants is presented for example in $[5,6,48,62]$. An infinite dimensional analogue for parabolic distributed parameter systems appeared in [21] and for hyperbolic distributed parameter systems in [9]. Using the linearity assumption $(D 1)$ with (5), the term $\beta\left(t-t^{*}\right) D(\theta) x(t)$ takes the form

$$
\begin{aligned}
\beta\left(t-t^{*}\right) D(\theta) x(t) & = \begin{cases}\left(1+\kappa \mathrm{e}^{-\alpha\left(t-t^{*}\right)}\right) D\left(\theta_{s s}\right) x(t) & \text { for } t \geq t^{*} \\
0 & \text { for } t<t^{*}\end{cases} \\
& =\left\{\begin{array}{ll}
D\left(\theta_{s s}\left[1+\kappa \mathrm{e}^{-\alpha\left(t-t^{*}\right)}\right]\right) x(t) & \text { for } t \geq t^{*} \\
0 & \text { for } t<t^{*}
\end{array},\right.
\end{aligned}
$$

or, equivalently the parameter $\theta(t)$ is given by

$$
\theta(t)= \begin{cases}\theta_{s s}\left[1+\kappa \mathrm{e}^{-\alpha\left(t-t^{*}\right)}\right] & \text { for } t \geq t^{*} \\ 0 & \text { for } t<t^{*}\end{cases}
$$

When the above definition of the parameter $\theta(t)$ is written as a differential equation, it yields, for $t \geq t^{*}$, the following

$$
\frac{\mathrm{d}}{\mathrm{d} t}\left(\theta(t)-\theta_{s s}\right)=-\alpha\left(\theta(t)-\theta_{s s}\right), \quad t \geq t^{*},
$$

or when we set $\phi(t)$ to be the difference of $\theta(t)$ from its steady state value $\theta_{s s}$, we have

$$
\frac{\mathrm{d}}{\mathrm{d} t} \phi(t)=-\alpha \phi(t), \quad t \geq t^{*}, \quad \phi\left(t^{*}\right)=\theta\left(t^{*}\right)-\theta_{s s}=\kappa \theta_{s s} .
$$

Equation (26) defines the class of plants with time varying parameters, namely plants whose parameters converge to their steady state value in an exponential fashion. The well-posedness of the plant after the failure, given here by

$$
\dot{x}(t)+\left[A+\beta\left(t-t^{*}\right) D\left(\theta_{s s}\right)\right] x(t)=B u(t), \quad x\left(t^{*}\right) \in H,
$$

with $\beta\left(t-t^{*}\right)=H\left(t-t^{*}\right)\left[1+\kappa e^{-\alpha\left(t-t^{*}\right)}\right]$, with $H(t)$ denoting the Heaviside step function, can be established in a similar fashion as the one presented in Section 2. Additional assumptions must be imposed. In this case, the inequality

$$
\mathcal{R} e\left\langle\left[A+\beta\left(t-t^{*}\right) D\left(\theta_{s s}\right)\right] \varphi, \varphi\right\rangle+\lambda_{a}|\varphi|^{2} \geq\left(\beta_{a}-\alpha_{d}\left|\beta\left(t-t^{*}\right)\right|_{\infty}\left|\theta_{s s}\right|_{Q}\right)\|\varphi\|^{2}
$$


must at least satisfy

$$
0<\beta_{a}-\alpha_{d}\left|\beta\left(t-t^{*}\right)\right|_{\infty}\left|\theta_{s s}\right|_{Q}=\beta_{a}-\alpha_{d}(1+|\kappa|)\left|\theta_{s s}\right|_{Q}
$$

which is a condition on both the steady state value $\theta_{s s}$ of the parameter $\theta(t)$ and the parameter $\kappa$.

The estimator equations are the same used for the time-invariant case, namely equations (11-13). The error equations will be different in the sense that the previously defined parameter error $r(t)$ will be written as

$$
r(t)=\widehat{\theta}(t)-\theta(t)=\left(\widehat{\theta}(t)-\theta_{s s}\right)+\left(\theta_{s s}-\theta(t)\right)=\zeta(t)-\phi(t),
$$

where $\zeta(t)=\widehat{\theta}(t)-\theta_{s s}$ and $\phi(t)$ is as defined in (26) above, see [9] for similar treatment of adaptive estimation of second order distributed parameter systems. Therefore, the error equations are written as

$$
\begin{gathered}
\dot{e}(t)+L e(t)+G^{*}(x(t))[\zeta(t)-\phi(t)]=0, \\
\dot{\zeta}(t)-G(x(t)) e(t)=0 .
\end{gathered}
$$

The arguments leading to the well posedness of the estimator are identical to those presented in Section 2 . The energy function now becomes

$$
\widetilde{E}(t)=\frac{1}{2}\left\{|e(t)|^{2}+|\zeta(t)|_{Q}^{2}+\nu|\phi(t)|_{Q}^{2}\right\}
$$

where $\nu$ is some positive constant to be defined below (see [48] for similar definition in the finite dimensional case). Using (26-28) and Definition 2.3, the time derivative of (29) is given by

$$
\begin{aligned}
\frac{\mathrm{d}}{\mathrm{d} t} \widetilde{E}(t)= & \left\langle\frac{\mathrm{d}}{\mathrm{d} t} e(t), e(t)\right\rangle+\left\langle\frac{\mathrm{d}}{\mathrm{d} t} \zeta(t), \zeta(t)\right\rangle_{Q}+\nu\left\langle\frac{\mathrm{d}}{\mathrm{d} t} \phi(t), \phi(t)\right\rangle_{Q}=-\langle L e(t), e(t)\rangle-\left\langle G^{*}(x(t))[\zeta(t)-\phi(t)], e(t)\right\rangle \\
& +\langle G(x(t)) e(t), \zeta(t)\rangle-\nu\langle\alpha \phi(t), \phi(t)\rangle_{Q} \\
= & -\langle L e(t), e(t)\rangle+\left\langle G^{*}(x(t)) \phi(t), e(t)\right\rangle-\alpha \nu\langle\phi(t), \phi(t)\rangle_{Q} \\
\leq & -\beta_{l}\|e(t)\|^{2}+\gamma\|e(t)\||\phi(t)|_{Q}-\alpha \nu|\phi(t)|_{Q}^{2} \\
\leq & -\left(\beta_{l}-\gamma \frac{\mu}{2}\right)\|e(t)\|^{2}-\left(\alpha \nu-\gamma \frac{1}{2 \mu}\right)|\phi(t)|_{Q}^{2} \\
= & -c_{1}\|e(t)\|^{2}-c_{2}|\phi(t)|_{Q}^{2}
\end{aligned}
$$

where $c_{1}=\beta_{l}-\gamma \frac{\mu}{2}$ and $c_{2}=\alpha \nu-\gamma \frac{1}{2 \mu}$ and $\mu$ is such that $c_{1}>0$ and $c_{2}>0$. The choice of the constant $\nu>\frac{\gamma^{2}}{4 \alpha \beta_{l}}$ with $\mu<\frac{2 \beta_{l}}{\gamma}$ will ensure that both $c_{1}$ and $c_{2}$ are strictly positive. It is worth mentioning that in the above calculations we made use of the following arithmetic-geometric mean inequality $a \cdot b \leq \frac{\mu}{2} a^{2}+\frac{1}{2 \mu} b^{2}$.

Remark 4.1. A somewhat different method of using an alternative expression of the Lyapunov functional appeared in the finite dimensional treatment of fault detection in [30]. There, the temporal variation of the parameter $\theta(t)$ was manipulated as an additional term in the definition of the Lyapunov functional.

Remark 4.2. It should be noted that the above extension to time varying systems is defined for the case of constant-in-space parameter $\theta$, i.e. no spatially varying functional parameters. The case of functional time varying parameters would require the equivalent Gelfand triple for the parameter space $Q$ as it is often the case that the differential operator that describes the time evolution of the functional parameter is unbounded. This is the subject of future study since the purpose of this note is to give a basic exposition to the fault detection schemes of infinite dimensional systems. In the event that the unknown parameter is a single time varying functional parameter that satisfies an equation of the form

$$
\frac{\mathrm{d}}{\mathrm{d} t}\left(\theta(t, \xi)-\theta_{s s}(\xi)\right)=-\alpha\left(\theta(t, \xi)-\theta_{s s}(\xi)\right)
$$


where $\xi$ is the spatial parameter in a bounded domain $\Omega$, and it is assumed that the initial condition $\theta\left(t^{*}, \xi\right)$ is a constant multiple of the steady state $\theta_{s s}(\xi)$ for all $\xi \in \Omega$, i.e. $\theta(t, \xi)$ is of the form

$$
\theta(t, \xi)= \begin{cases}\theta_{s s}(\xi)\left[1+\kappa \mathrm{e}^{-\alpha\left(t-t^{*}\right)}\right] & \text { if } t \geq t^{*} \\ 0 & \text { if } t<t^{*}\end{cases}
$$

with $\theta\left(t^{*}, \xi\right)=(1+\kappa) \theta_{s s}(\xi)$ for all $\xi \in \Omega$, then the above proposed adaptation scheme can still be employed to detect failures in the infinite dimensional system $(7,8)$ with both temporally (slowly time varying) and spatially varying parameters.

Remark 4.3. It might appear, due to equation (25), that the parameter $\theta$ is only a single scalar time varying parameter. In fact the above extension is also valid in the case where the parameter $\theta$ is a vector (denoted by $\vec{\theta})$ that satisfies a differential equation of the form

$$
\frac{\mathrm{d}}{\mathrm{d} t}\left(\vec{\theta}(t)-\vec{\theta}_{s s}\right)=\Lambda\left(\vec{\theta}(t)-\vec{\theta}_{s s}\right)
$$

where the matrix $\Lambda$ is a stable matrix with diagonal elements. It might be the case that the matrix $\Lambda$ is not diagonal and still the above would hold. In this case we must impose the condition that $\Lambda$ is diagonalizable and that by the appropriate weighting of the $Q$-inner product we can have a diagonal matrix in (31). For the sake of simplicity, we used equation (25) instead of (31), but all the calculations presented above are still valid in the vector case.

The same convergence results presented in Section 3 can be used in the case of time varying parameters. The corresponding lemma to Lemma 3.1 is

Lemma 4.4. For all $t>t^{*}$ we have

$$
\widetilde{E}(t)+c_{1} \int_{t^{*}}^{t}\|e(\tau)\|^{2} \mathrm{~d} \tau+c_{2} \int_{t^{*}}^{t}|\phi(\tau)|_{Q}^{2} \mathrm{~d} \tau \leq E\left(t^{*}\right) .
$$

Identical convergence results are obtained in this case, with the exception that Theorem 3.2 applies to the error equations $(27,28)$.

\section{EXAMPleS}

In this section we present some examples to demonstrate the applicability of the proposed fault detection scheme. We first examine a one dimensional heat equation with constant coefficients and then a one dimensional heat equation with time varying parameters. In addition to these examples, we also examine a second order (in time) hyperbolic pde (wave equation) with spatially varying fault of the stiffness parameter and then a nonlinear parabolic pde in which the fault is modeled as a nonlinear perturbation of the thermal diffusivity involving a function of the heat flux.

All computations are carried out by a numerical approximation method using finite element methods with the linear spline elements [7,16,57] and the Fehlberg fourth-fifth order Runge-Kutta method for time integration [58]. The numerical implementation of the proposed learning scheme and its finite dimensional approximation and convergence proofs will appear in greater detail in a forthcoming paper, see [20] for similar results on the adaptive parameter identification of abstract parabolic and hyperbolic distributed parameter systems.

\subsection{Example 1}

As a first example, we consider the one dimensional diffusion equation with spatially varying parameter given by

$$
\frac{\partial}{\partial t} x(t, \xi)=\frac{\partial}{\partial \xi}\left(a(\xi) \frac{\partial}{\partial \xi} x(t, \xi)\right)+f(t, \xi)+\beta(t-10) \frac{\partial}{\partial \xi}\left(\theta(\xi) \frac{\partial}{\partial \xi} x(t, \xi)\right)
$$


and with boundary and initial conditions $x(t, 0)=x(t, 1)=0, t>0, x(0, \xi)=0,0<\xi<1$. The Hilbert space $H$ is taken to be $H=L_{2}(0,1)$ and the Sobolev space $V$ is $V=H_{0}^{1}(0,1)$; the reader is directed to the books of Adams [3] or Lions and Magenes [45] for an exposition to Sobolev spaces. The parameter space in this case is $Q=H^{1}(0,1)$ endowed with the weighted inner product

$$
\langle q, p\rangle_{Q}=\omega_{1} \int_{0}^{1} q(\xi) \cdot p(\xi) \mathrm{d} \xi+\omega_{2} \int_{0}^{1} q^{\prime}(\xi) \cdot p^{\prime}(\xi) \mathrm{d} \xi, \quad q, p \in H^{1}(0,1),
$$

where the weights $\omega_{1}$ and $\omega_{2}$ are assumed to be positive. The operators $A$ and $D(\cdot)$ in (1) are given by

$$
\langle A \phi, \psi\rangle=\int_{0}^{1} a(\xi) \cdot \phi^{\prime}(\xi) \cdot \psi^{\prime}(\xi) \mathrm{d} \xi, \quad\langle D(\theta) \phi, \psi\rangle=\int_{0}^{1} \theta(\xi) \cdot \phi^{\prime}(\xi) \cdot \psi^{\prime}(\xi) \mathrm{d} \xi
$$

The operator $G(x(t))$ in (5) is given in weak form by

$$
\langle G(x(t)) \varphi, p\rangle_{H^{1}(0,1)}=\langle D(p) x(t), \varphi\rangle=\int_{0}^{1} p(\xi) \frac{\partial}{\partial \xi} x(t, \xi) \cdot \varphi^{\prime}(\xi) \mathrm{d} \xi,
$$

for $\varphi \in H_{0}^{1}(0,1), p \in H^{1}(0,1)$, and the estimator operator $L$ is given by

$$
\langle L \phi, \psi\rangle=2 \int_{0}^{1} \phi(\xi) \cdot \psi(\xi) \mathrm{d} \xi, \quad \phi, \psi \in H_{0}^{1}(0, l) .
$$

In this case, the effect of the fault is a change in the thermal diffusivity from $a(\xi)$ to $a(\xi)+\theta(\xi)$. In other words,

$$
\left.D(\theta)\right|_{\theta(\xi)=a(\xi)} x(t) \equiv A x(t)
$$

or the $\theta$-parameterized operator $D(\theta)$ is the same as the nominal operator $A$ evaluated at a different diffusivity parameter. The thermal diffusivity is given by $a(\xi)=1.5 \times 10^{-3}(1.5-\sin (\pi \xi)), 0 \leq \xi \leq 1$, and the unknown perturbation $\theta(\xi)$ of the diffusivity is chosen as

$$
\theta(\xi)=1.5 \times 10^{-3}\left(1-\frac{\sqrt{2}}{2}-\sin (3 \pi \xi)\right) \chi_{[0.3,0.7]}(\xi), \quad 0 \leq \xi \leq 1,
$$

where $\chi_{[0.3,0.7]}$ denotes the characteristic function over the interval $[0.3,0.7]$. This is also illustrated in Figure 1 where both $a(\xi)$ and $a(\xi)+\theta(\xi)$ are depicted. It can be easily verified that assumptions $(D 1),(D 2),(A 1),(A 2)$ and $(L 1),(L 2)$ are satisfied with $\alpha_{d}=1.0, \lambda_{a}=0, \alpha_{a}=2.25 \times 10^{-3}, \beta_{a}=0.75 \times 10^{-3}, \alpha_{l}=\beta_{l}=2$. The embedding constant in (4) is $K=\pi^{-1}$. It follows that

$$
\langle[A+D(\theta)] \varphi, \varphi\rangle=\int_{0}^{1}(a(\xi)-\theta(\xi))\left[\varphi_{\xi}(\xi)\right]^{2} \mathrm{~d} \xi \geq 1 \times 10^{-3}\|\varphi\|^{2}
$$

will guarantee the existence of a unique solution as mentioned in Section 2. The forcing function is given by

$$
f(t, \xi)=10^{-2}\left\{\left[3+0.1 \sin \left(\frac{\pi t}{50}\right)\right] \times 2 \mathrm{e}^{-0.01 t}+\sin \left(\frac{\pi t}{200}\right)\right\} \chi_{[0.3,0.7]}(\xi) .
$$

In Figure 2, we observe that both $|e(t)|$ and $|r(t)|$ remain at zero for $0 \leq t<t^{*}$. The estimated model approximates the system after failure and the state increases (sudden jump) at the time of the system failure at $t=10$ seconds but converges to zero within 6 seconds. Additionally, we observe that the on-line parameter 

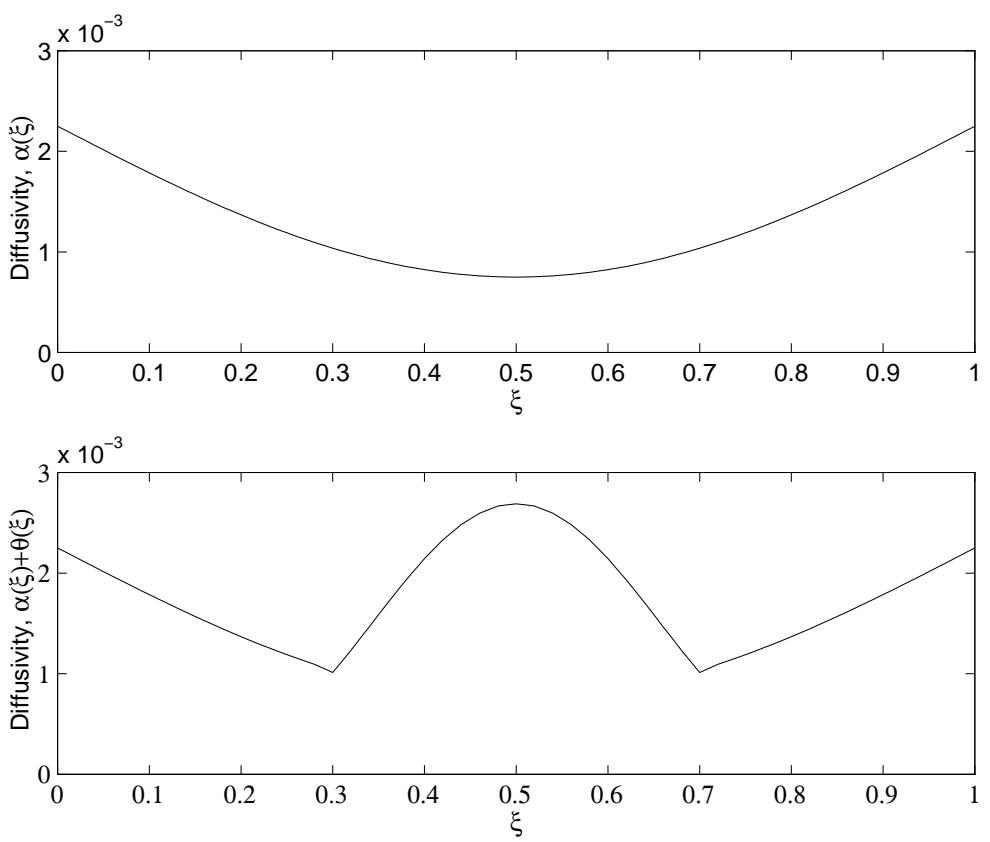

FiguRE 1. Example 1; Diffusivity parameter $\alpha(\xi)$ before failure and $\alpha(\xi)+\theta(\xi)$ after failure.

approximator can also serve as an indicator of the system's failure. The large magnitudes of these jumps can be attributed to the large values of both the coercivity of the operator $L$ and the adaptive gains (reciprocals of the weights of the inner-product of the parameter space). Figure 3 shows the parameter approximator $\widehat{\theta}(t, \xi)$ compared to the actual parameter $\theta(\xi)$ at four different time instances. It is observed that the parameter approximator identifies the location (i.e. function is nonzero on the spatial interval $0.3 \leq \xi \leq 0.7$ ) and the shape of the failure (i.e. the function $1-\frac{\sqrt{2}}{2}-\sin (3 \pi \xi)$ ) within 4 seconds of the failure occurrence, from $t=11$ seconds to $t=14$ seconds. It should be noted that smaller value of $L$ would result in a prolonged diagnosis.

\subsection{Example 2}

In the next example, we consider a heat equation of the form

$$
\frac{\partial}{\partial t} x(t, \xi)=\frac{\partial}{\partial \xi}\left(a(\xi) \frac{\partial}{\partial \xi} x(t, \xi)\right)+f(t, \xi)+\beta(t-10) \theta(t) x(t, \xi),
$$

with boundary and initial conditions given by $x(t, 0)=x(t, 1)=0, t>0, x(0, \xi)=0,0 \leq \xi \leq 1$. Once again we set $H=L_{2}(0,1)$ and $V=H_{0}^{1}(0,1)$ endowed with the usual inner products and corresponding induced norms, with the parameter space now given by $Q=\mathbb{R}^{1}$. We endowed the parameter space with the weighted Euclidean inner product $\langle q, p\rangle_{Q}=\omega p q, q, p \in \mathbb{R}^{1}$ where the weight $\omega>0$. The embedding constant $K=\pi^{-1}$ (see, for example $[7,57])$. The operator $A$ in (1) and the estimator operator $L$ in (11) are given by

$$
\langle A \phi, \psi\rangle=\int_{0}^{1} a(\xi) \cdot \phi^{\prime}(\xi) \cdot \psi^{\prime}(\xi) \mathrm{d} \xi, \quad\langle L \phi, \psi\rangle=2 \int_{0}^{1} \phi(\xi) \cdot \psi(\xi) \mathrm{d} \xi,
$$



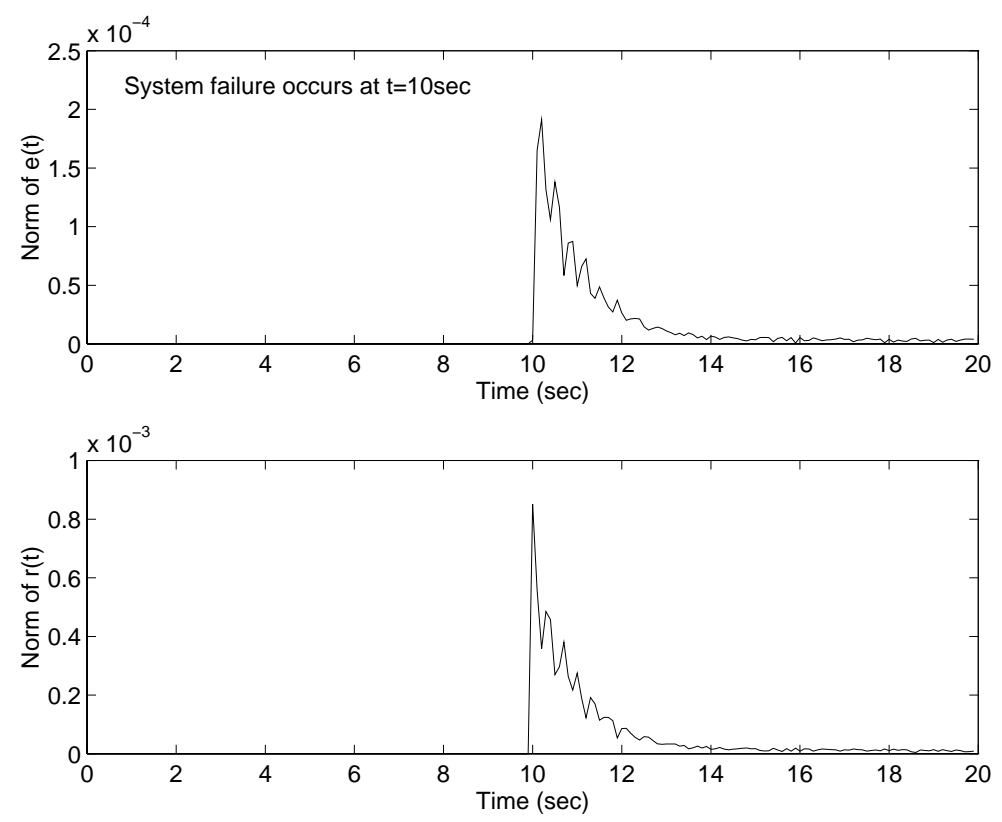

Figure 2. Example 1; Evolution of state error, $e(t)=\widehat{x}(t)-x(t)$, and parameter error $r(t)=$ $\widehat{\theta}(t)-\theta$.

for $\phi, \psi \in H_{0}^{1}(0,1)$, and for $\theta \in Q$, the operator $D(\theta)$ is given by

$$
\langle D(\theta(t)) \phi, \psi\rangle=-\theta(t) \int_{0}^{1} \phi(\xi) \cdot \psi(\xi) \mathrm{d} \xi, \quad \phi, \psi \in H_{0}^{1}(0,1)
$$

with the parameter $\theta$, defined for $t \geq 10$, given by

$$
\theta(t)=0.01\left(1+3 \mathrm{e}^{-0.02(t-10)}\right), \quad \theta_{s s}=0.01, \quad \theta(10)=0.04
$$

The nominal plant parameter (i.e. the diffusivity parameter $\alpha(\xi))$ is given by $\alpha(\xi)=1.5 \times 10^{-2}\left\{1-\frac{1}{2} \sin \left(2 \pi\left[\xi-\frac{1}{4}\right]\right)\right\}$, $0 \leq \xi \leq 1$. The forcing function is given by

$$
f(t, \xi)=10^{-2}\left[3+0.1 \sin \left(\frac{\pi t}{50}\right)\right] \times 2 \mathrm{e}^{-0.01 t} \chi_{[0.3,0.7]}(\xi)
$$

Using the above, we can easily verify again that assumptions $(D 1),(D 2),(A 1),(A 2),(L 1),(L 2)$ are satisfied with $\alpha_{d}=1 / \pi^{2}, \alpha_{a}=2.25 \times 10^{-2}, \lambda_{a}=0, \beta_{a}=7.5 \times 10^{-3}$, and $\alpha_{l}=\beta_{l}=2$. In addition, we see that

$$
\begin{aligned}
\langle[A+D(\theta(t))] \varphi, \varphi\rangle & =\int_{0}^{1} a(\xi)\left[\varphi_{\xi}(\xi)\right]^{2}-\theta(t)[\varphi(\xi)]^{2} \mathrm{~d} \xi \geq\left(\beta_{a}-\alpha_{d}(1+|\kappa|) \theta_{s s}\right) \int_{0}^{1}\left[\varphi_{\xi}(\xi)\right]^{2} \mathrm{~d} \xi \\
& \geq 3.4 \times 10^{-3}\|\varphi\|^{2}
\end{aligned}
$$

which guarantees the existence of a unique solution to (33). The estimator in this case is given by

$$
\frac{\partial}{\partial t} \widehat{x}(t, \xi)=\frac{\partial}{\partial \xi}\left(a(\xi) \frac{\partial}{\partial \xi} x(t, \xi)\right)+f(t, \xi)-2[\widehat{x}(t, \xi)-x(t, \xi)]+\widehat{\theta}(t) x(t, \xi)
$$



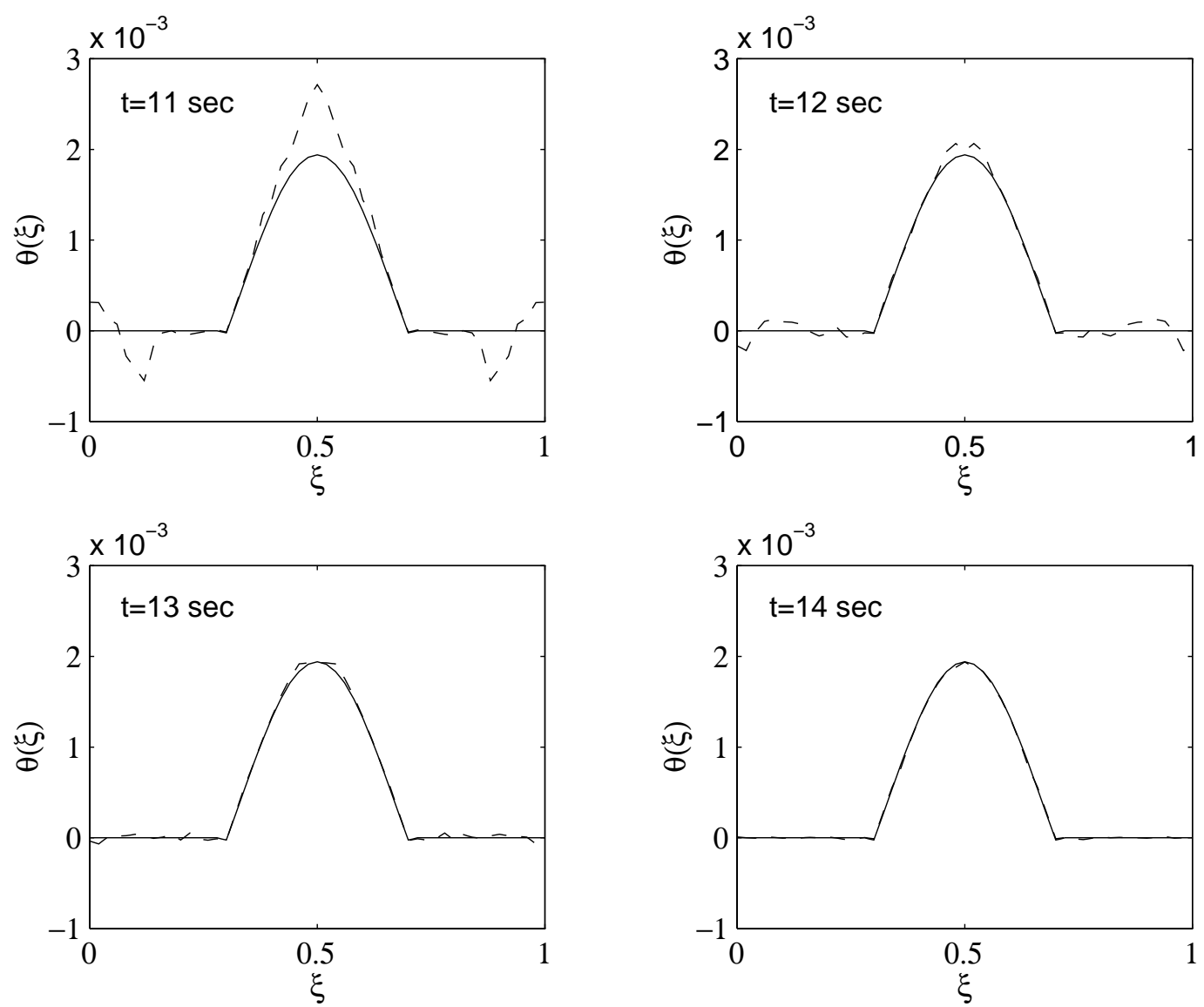

Figure 3. Example 1; Parameter $\hat{\theta}(t, \xi)$ (dashed) and $\theta(\xi)$ (solid) at different time epochs $t=11,12,13 \& t=14$ seconds.

and the parameter adaptation rule is given by

$$
\dot{\hat{\theta}}(t)=-\gamma\langle e(t), x(t)\rangle=-\gamma \int_{0}^{1}[\widehat{x}(t, \xi)-x(t, \xi)] \cdot x(t, \xi) \mathrm{d} \xi, \quad \widehat{\theta}(0)=0
$$

In this case the adaptive gain $[43,48]$ is $\gamma=\frac{1}{\omega}$ and is chosen to be $\gamma=100$.

In a similar way as in the previous example, it can be observed from Figure 4 that both $|e(t)|$ and $|r(t)|$ remain at zero for $t<10$. Both become nonzero for $t \geq 10$ and eventually converge to zero within 6 seconds. A natural question that arises is whether $\widehat{\theta}(t)$ converges to $\theta(t)$ before $\theta(t)$ converges to its steady state value $\theta_{s s}$, or while $\theta(t)$ is moving towards $\theta_{s s}$. This is depicted in Figure 5 , where we see that $\widehat{\theta}(t)$ converges to $\theta(t)$ around the $16^{t h}$ second (Fig. 5b) whereas $\theta(t)$ converges to its steady state value $\theta_{s s}$ after $t>100$ seconds. The parameter convergence theory (via the persistence of excitation) in Section 4 does not provide any answer as to whether the parameter estimate will converge to $\theta_{s s}$ or to $\theta(t)$ before the latter converges to $\theta_{s s}$. This certainly would depend on the rate of convergence $\alpha$ of $\theta(t)$ to $\theta_{s s}$ and the level of persistence of excitation. This is an issue still under investigation by the author. 

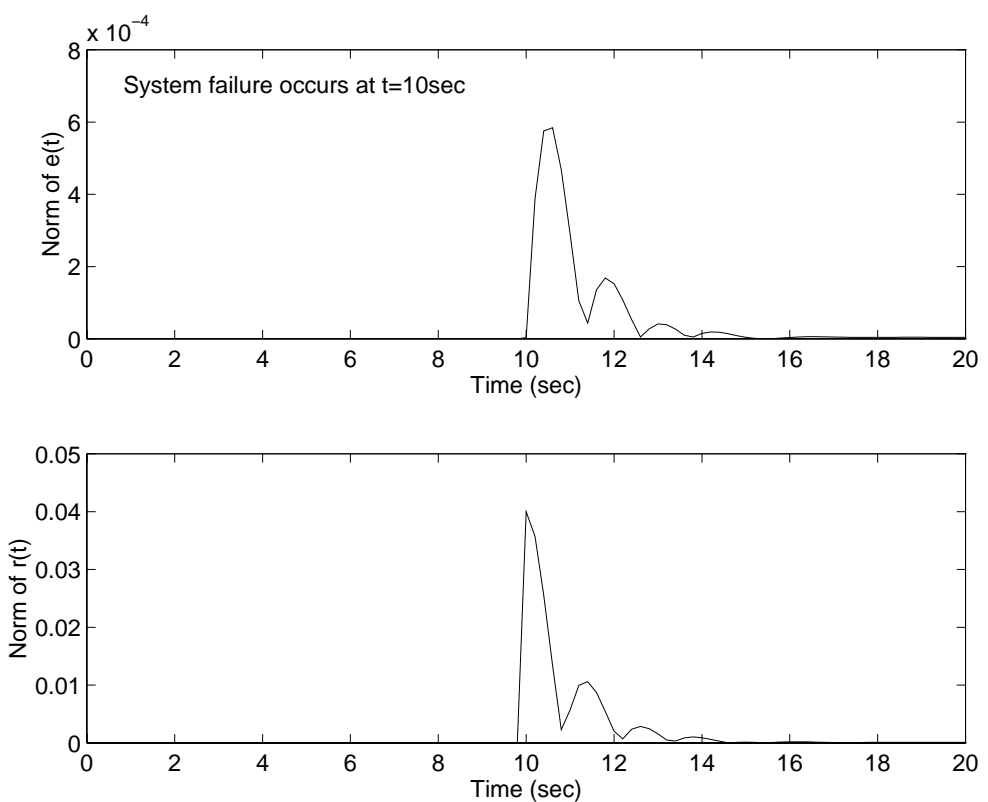

Figure 4. Example 2; Evolution of state error, $e(t)=\widehat{x}(t)-x(t)$, and parameter error $r(t)=$ $\widehat{\theta}(t)-\theta(t)$.

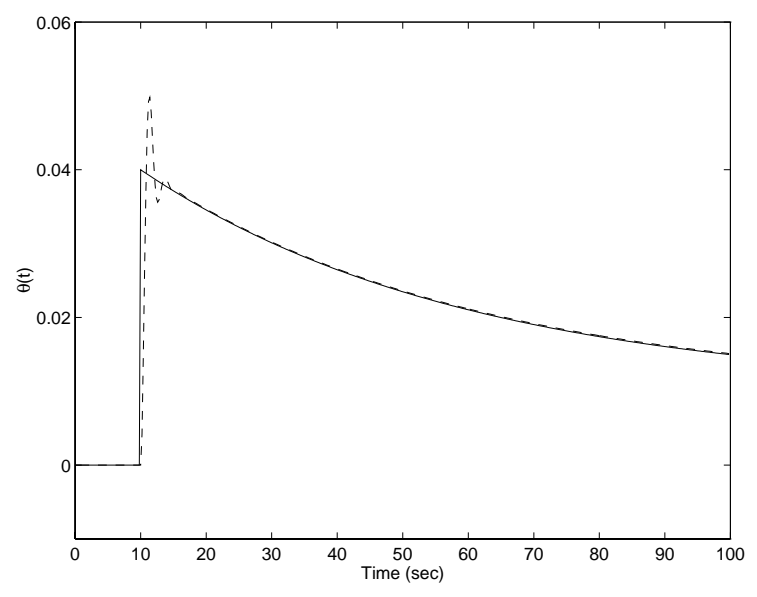

(a)

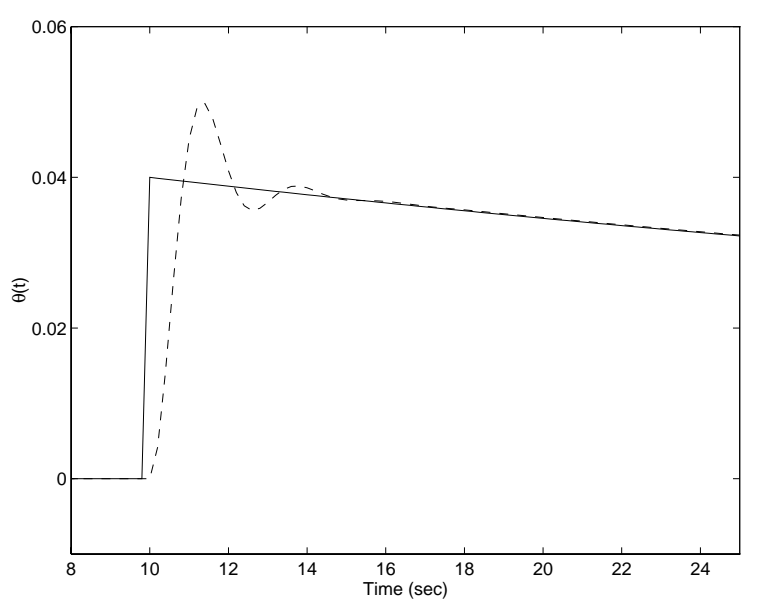

(b)

Figure 5. Example 2; Parameter $\widehat{\theta}(t)$ (dashed) and $\theta(t)$ (solid); (a) time interval [0, 100], (b) enlarged time window $[8,25]$.

\subsection{Example 3}

In this example we consider a second order system that can be written as a first order system. The plant is given by the wave equation with Kelvin-Voigt viscoelastic damping

$$
\frac{\partial^{2}}{\partial t^{2}} w(t, \xi)-\frac{\partial}{\partial \xi}\left(c_{D} I(\xi) \frac{\partial^{2}}{\partial \xi \partial t} w(t, \xi)+E I(\xi) \frac{\partial}{\partial \xi} w(t, \xi)\right)-\beta(t-10) \frac{\partial}{\partial \xi}\left(\theta(\xi) \frac{\partial}{\partial \xi} w(t, \xi)\right)=f(t, \xi), \quad \text { in } \Omega
$$


where $w(t, \xi)$ denotes the displacement and $w_{t}(t, \xi)$ the velocity. The boundary and initial conditions are given by $\left.w(t, \xi)\right|_{\partial \Omega}=\left.w_{x}(t, \xi)\right|_{\partial \Omega}=0, w(0, \xi)=d_{0}(\xi) \in H_{0}^{1}(0, l), w_{t}(0, \xi)=v_{0}(\xi) \in L^{2}(0, l)$. Since equation (34) has strong damping, we can use the same techniques in [20] applied for the adaptive parameter estimation of hyperbolic distributed parameter systems, to write equation (34) as a first order system with $H=H_{0}^{1}(0, l) \times$ $L^{2}(0, l), V=H_{0}^{1}(0, l) \times H_{0}^{1}(0, l)$ and the parameter space $Q=H^{1}(0, l)$. We briefly describe the procedure. When the above system is written as an abstract second order initial value problem, we arrive at

$$
w_{t t}(t)+K_{1} w_{t}(t)+K_{2} w(t)+\beta(t-10) K_{3}(\theta) w(t)=f(t), \text { a.e. } t>0
$$

with $w(0)=d_{0}, w_{t}(0)=v_{0}$, which when written in a weak (or variational) form becomes

$$
\left\langle w_{t t}(t), \phi\right\rangle+\left\langle K_{1} w_{t}(t), \phi\right\rangle+\left\langle K_{2} w(t), \phi\right\rangle+\beta(t-10)\left\langle K_{3}(\theta) w(t), \phi\right\rangle=\langle f(t), \phi\rangle,
$$

$w(0)=d_{0} \in H_{0}^{1}(0, l), w_{t}(0)=v_{0} \in L^{2}(0, l)$. The state and parameter estimators are given, for $\phi=\left(\phi_{1}, \phi_{2}\right) \in$ $H_{0}^{1}(0, l) \times L^{2}(0, l)$, by

$$
\begin{aligned}
& \left\langle L_{2} \frac{\mathrm{d}}{\mathrm{d} t} \widehat{w}(t), \phi_{1}\right\rangle=\left\langle L_{2} \widehat{w}_{t}(t), \phi_{1}\right\rangle+\lambda\left\langle L_{2} e(t), \phi_{1}\right\rangle \\
& \left\langle\frac{\mathrm{d}}{\mathrm{d} t} \widehat{w}_{t}(t), \phi_{2}\right\rangle+\left\langle L_{1} e_{t}(t), \phi_{2}\right\rangle+\left\langle L_{2} e(t), \phi_{2}\right\rangle+\lambda\left\langle e_{t}(t), \phi_{2}\right\rangle+\left\langle K_{3}(\widehat{\theta}(t)) w(t), \phi_{2}\right\rangle=\left\langle f(t), \phi_{2}\right\rangle
\end{aligned}
$$

and

$$
\left\langle\frac{\mathrm{d}}{\mathrm{d} t} \widehat{\theta}(t), p\right\rangle_{H^{1}(0, l)}=\left\langle K_{3}(p) w(t), e_{t}(t)\right\rangle, p \in H^{1}(0, l) .
$$

The design operators $L_{1}, L_{2} \in \mathcal{L}\left(H_{0}^{1}(0, l), H^{-1}(0, l)\right)$ are chosen to satisfy assumptions $(L 1)$ and $(L 2)$ and the operators $K_{i} \in \mathcal{L}\left(H_{0}^{1}(0, l), H^{-1}(0, l)\right), i=1,2, K_{3}(\theta) \in \mathcal{L}\left(H_{0}^{1}(0, l), H^{-1}(0, l)\right), \theta \in Q$, are given by

$$
\begin{aligned}
\left\langle K_{1} w_{t}(t), \psi\right\rangle & =\int_{0}^{l} c_{D} I(\xi) \cdot w_{t}^{\prime}(t, \xi) \cdot \psi^{\prime}(\xi) \mathrm{d} \xi \\
\left\langle K_{2} w(t), \psi\right\rangle & =\int_{0}^{l} E I(\xi) \cdot w^{\prime}(t, \xi) \cdot \psi^{\prime}(\xi) \mathrm{d} \xi \\
\left\langle K_{3}(\theta) w(t), \psi\right\rangle & =\int_{0}^{l} \theta(\xi) \cdot w^{\prime}(t, \xi) \cdot \psi^{\prime}(\xi) \mathrm{d} \xi
\end{aligned}
$$

The fault is modeled as a change (decrease) in the stiffness parameter $E I$ which is given below and depicted in Figure 6,

$$
\theta(\xi)=-1.5 \times 10^{-4} \chi_{[0.3,0.7]}(\xi)\left(1-\frac{\sqrt{2}}{2}-\sin (3 \pi \xi)\right), 0<\xi<l .
$$

Since in this case $E I(\xi)+\theta(\xi)>0 \forall \xi \in[0, l]$ and hence $\left\langle\left(K_{1}+K_{3}(\theta)\right) \varphi, \varphi\right\rangle \geq \alpha\|\varphi\|^{2}$, we can conclude well posedness using already established results on second order systems.

The stiffness and damping parameters $E I(\xi)$ and $c_{D} I(\xi)$ are chosen as $E I(\xi)=3 \times 10^{-2}$ and $c_{D} I(\xi)=$ $5 \times 10^{-3}, 0<\xi<l$ respectively. The input $f(t, \xi)$ is given by

$$
f(t, \xi)=\chi_{[0.4,0.6]}(\xi)\left(\sin \left(\frac{\pi t}{200}\right)+5\left(3+0.1 \sin \left(\frac{\pi t}{50}\right)\right) \mathrm{e}^{-0.01 t}\right) .
$$

The constant $\lambda$ is set to $\lambda=1$ and the initial conditions $d_{0}(\xi), v_{0}(\xi)$ are given by $d_{0}(\xi)=0.01 \sin (\pi \xi / l)$, $v_{0}(\xi)=0.001 \sin (4 \pi \xi / l), 0<\xi<l$. The design operators $L_{1}, L_{2}$ are chosen to have the same structure as the 

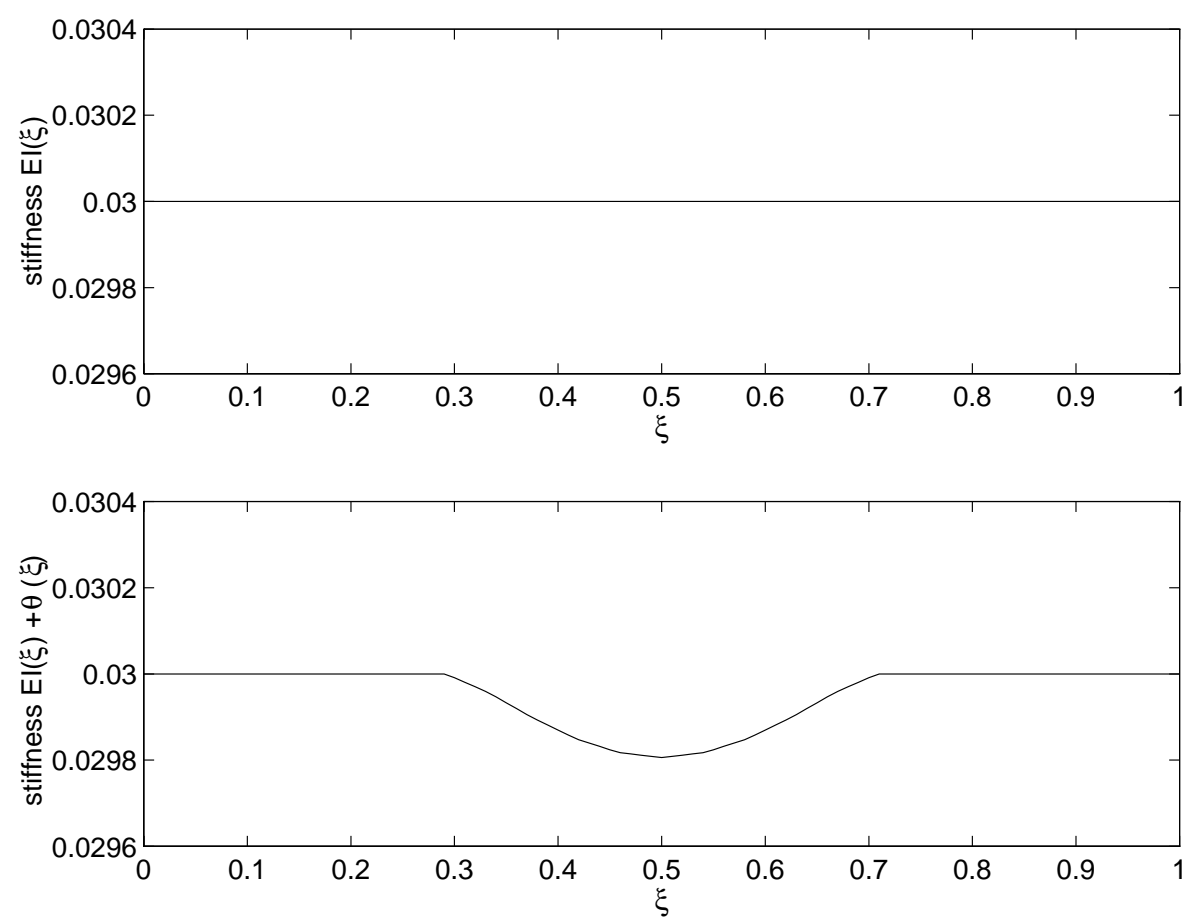

Figure 6. Example 3; Pre-damage stiffness parameter $E I(\xi)$ and post-damage stiffness $E I(\xi)+\theta(\xi)$.

damping and stiffness operators $K_{1}, K_{2}$ evaluated at different (and constant) damping and stiffness parameters, and are given by

$$
\left\langle L_{1} w_{t}(t), \psi\right\rangle=2 \times 10^{-2} \int_{0}^{l} w_{t \xi}(t, \xi) \cdot \psi^{\prime}(\xi) \mathrm{d} \xi, \quad\left\langle L_{2} w(t), \psi\right\rangle=4 \times 10^{-2} \int_{0}^{l} w_{\xi}(t, \xi) \cdot \psi^{\prime}(\xi) \mathrm{d} \xi
$$

for $\psi \in H_{0}^{1}(0, l)$. The adaptation rule for $\theta(t, \xi)$ is given by

$$
\left\langle\frac{\mathrm{d}}{\mathrm{d} t} \theta(t), p\right\rangle_{H^{1}(0, l)}=\int_{0}^{l} p(\xi) w_{\xi}(t, \xi) \cdot e_{t \xi}(t, \xi) \mathrm{d} \xi, \quad p \in H^{1}(0, l) .
$$

Both displacement and velocity state errors (in their respective norms) converge to zero after the failure occurs as depicted in Figure 7. The norm of the parameter estimate $\theta(t, \xi)$ converges to the norm of the actual parameter $\theta(\xi)$ as observed in Figure 8. The graph of the parameter estimate and its adaptive estimate are plotted (pointwise) in Figure 9. The pointwise convergence is established at 100 seconds.

\subsection{Example 4}

In the last example we consider a heat equation with the fault modeled as a nonlinear thermal conductivity. Specifically, the plant is given by

$$
\frac{\partial}{\partial t} u(t, \xi)=\frac{\partial}{\partial \xi}\left(a_{p} u_{\xi}(t, \xi)\right)+\beta(t-10) \frac{\partial}{\partial \xi}\left(\theta\left(u_{\xi}\right) u_{\xi}(t, \xi)\right)+f(t, \xi)
$$



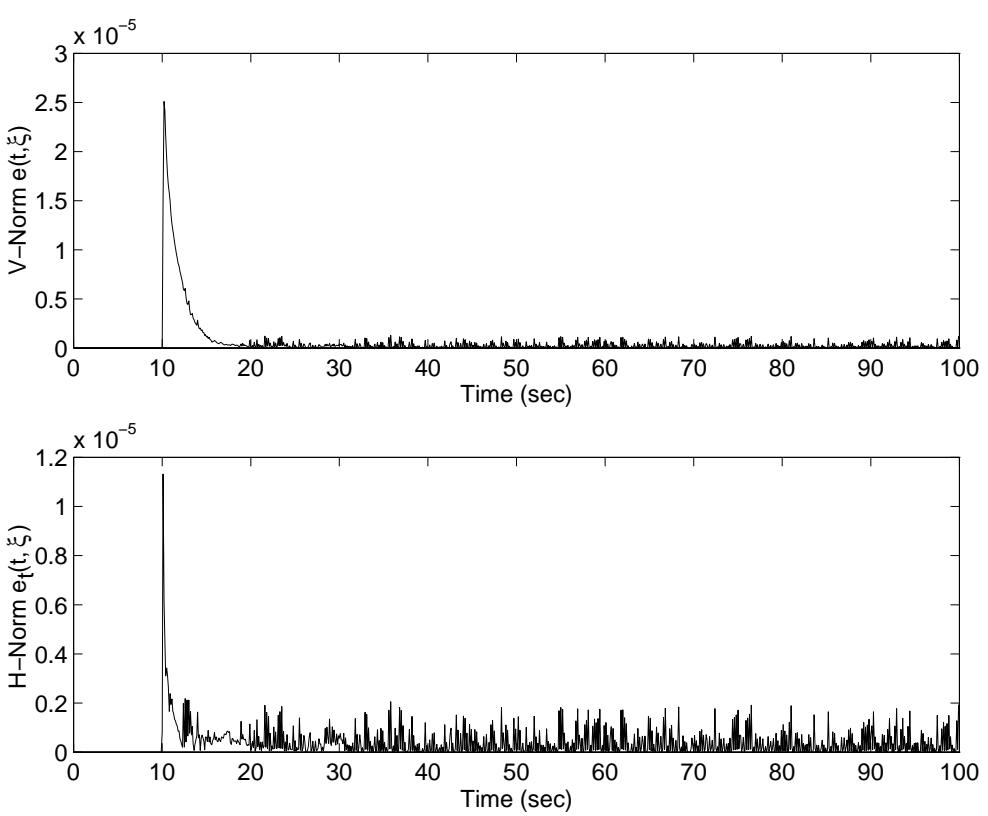

FiguRE 7. Example 3; Evolution of displacement state error $e(t, \xi)$ and velocity state error $e_{t}(t, \xi)$.
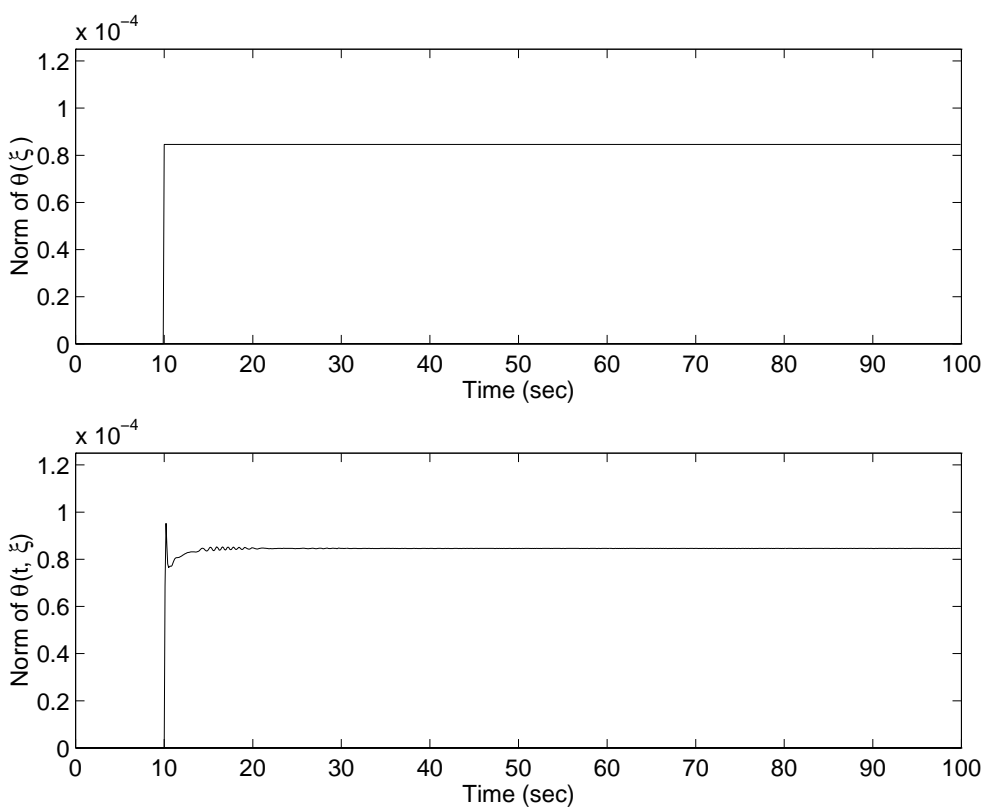

Figure 8. Example 3; Evolution of the parameter norm of $\beta(t-T) \theta(\xi)$ and its adaptive estimate $\widehat{\theta}(t, \xi)$.

for $0<\xi<1$ and $t>0$ together with the Dirichlet boundary conditions $u(t, 0)=u(t, 1)=1, t>0$, and initial condition $u(0, \xi)=u_{0}(\xi), 0<\xi<1$. The thermal conductivity is a function of the heat flux $u_{\xi}(t, \xi)$ and is given by $\theta(r)=0.9\left(1-0.5 e^{-0.5 * r^{2}}\right), r>0$. It is assumed that the initial condition $u_{0} \in L_{2}(0,1)$ and that 


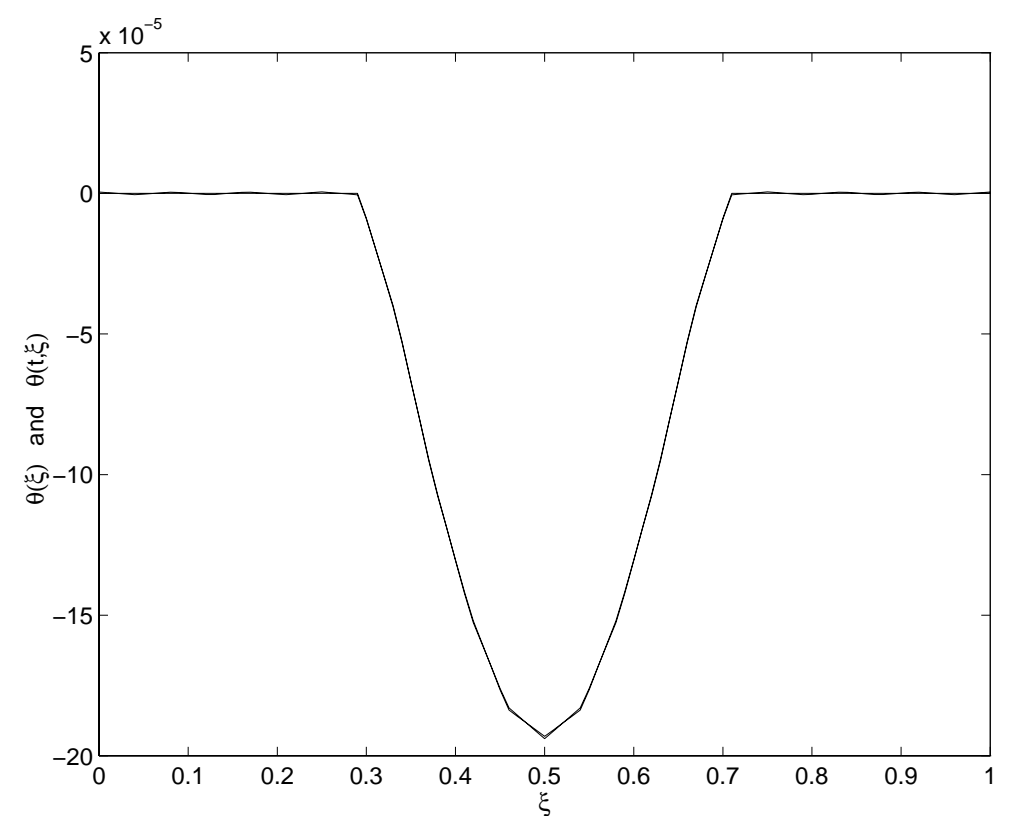

Figure 9. Example 3; Parameter $\theta(\xi)$ (dashed) and its adaptive estimate $\widehat{\theta}(t, \xi)$ (solid) at $t=100 \mathrm{sec}$ for $0<\xi<l$.

the input $f(t, \cdot) \in H^{-1}(0,1)$ for $t \geq 0$. The state space $H=L_{2}(0,1)$ with the standard inner product and the reflexive Banach space of test functions is taken to be $V=H_{0}^{1}(0,1)$. Unlike the previous examples, the Hilbert space $Q$ is defined as follows, see [13] for additional details regarding the setup of this specific example. Let $\widehat{Q}=\left\{\phi: \phi \in H_{l o c}^{1}\left(\mathbb{R}^{+}\right)\right.$and $\left.\phi, \phi^{\prime} \in L_{\infty}\left(\mathbb{R}^{+}\right)\right\}$. The inner product $\langle\cdot, \cdot\rangle_{Q}$ on $\widehat{Q}$ is defined by

$$
\langle\phi, \psi\rangle_{Q}=\int_{0}^{\infty} \omega_{0}(r) \phi(r) \psi(r) \mathrm{d} r+\int_{0}^{\infty} \omega_{1}(r) \phi^{\prime}(r) \psi^{\prime}(r) \mathrm{d} r, \quad \phi, \psi \in \widehat{Q}
$$

with $\omega_{0}, \omega_{1} \in L_{\infty}\left(\mathbb{R}^{+}\right)$positive weight functions. The Hilbert space $Q$ is defined as the completion of the inner product space $\left\{\widehat{Q},\langle\cdot, \cdot\rangle_{Q},|\cdot|_{Q}\right\}$, where $|\cdot|_{Q}$ is the norm induced by the inner product $\langle\cdot, \cdot\rangle_{Q}$. For $\theta \in Q$ we define the operator $D(\theta): V \rightarrow V^{*}$ by

$$
\langle D(\theta) \phi, \psi\rangle=\int_{0}^{1} \theta\left(\left|\phi^{\prime}\right|\right) \phi^{\prime}(\xi) \cdot \psi^{\prime}(\xi) \mathrm{d} \xi, \quad \phi, \psi \in H_{0}^{1}(0,1) .
$$

The nominal plant operator $A: V \rightarrow V^{*}$ and estimator operator $L$ are given by

$$
\langle A \phi, \psi\rangle=\int_{0}^{1} a_{p} \phi^{\prime}(\xi) \cdot \psi^{\prime}(\xi) \mathrm{d} \xi, \quad\langle L \phi, \psi\rangle=\int_{0}^{1} a_{m} \phi^{\prime}(\xi) \cdot \psi^{\prime}(\xi) \mathrm{d} \xi
$$

for $\phi, \psi \in H_{0}^{1}(0,1)$. The adaptation law for the parameter estimate is given by

$$
\langle\dot{\theta}(r), q\rangle_{Q}=-\int_{0}^{1} q\left(\left|u^{\prime}(t, \xi)\right|\right) u^{\prime}(t, \xi)\left[\widehat{u}^{\prime}(t, \xi)-u^{\prime}(t, \xi)\right] \mathrm{d} \xi, t>0,
$$

for $q \in Q$. Both norms of the state error $e$ and the parameter estimate $\widehat{\theta}$ are depicted in Figure 10, where we observe that they attain a nonzero value at the failure time $t^{*}=10$ seconds. The (norm of the) state error 

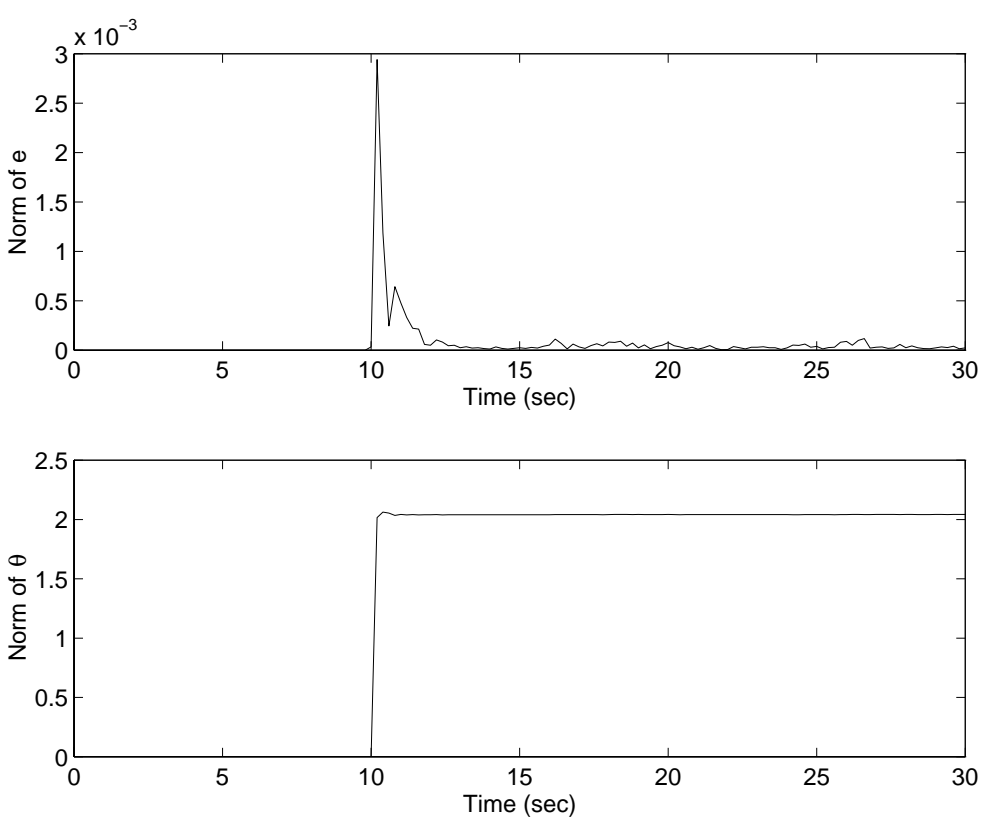

Figure 10. Example 4; Evolution of the state error $e(t, \xi)$ and the adaptive estimate $\widehat{\theta}$.

converges to zero within 5 seconds of the failure occurrence and the parameter norm remains at a fixed value. The latter does not provide any specific insight of the pointwise behavior of $\widehat{\theta}$. Figure 11 compares the function $\theta$ and its estimate for the time instance $t=30$ where the parameter convergence is observed.

Using the results in the above example, it should be observed that the proposed on-line fault detection scheme provided reasonable results in systems whose parameters appear linearly albeit themselves are nonlinear functions of the state.

\section{Conclusions and further Research}

In this note the finite dimensional theory of model-based fault diagnosis was extended into a class of infinite dimensional dynamical systems. The proposed state estimator (adaptive detection/diagnostic observer) with the parameter identifiers can detect the failure time. These on-line parameter estimators can identify (isolate) the location of the fault (in the spatial domain) and assess the nature of the fault thus allowing for the successful design of a control policy to accommodate such a system. The proposed scheme was designed with the applications for flexible structures in mind, but this general framework encompass systems governed by parabolic and hyperbolic partial differential equations. Delay differential equations can also be included in the proposed framework.

This scheme is by no means complete as it requires full state measurements, often impossible to acquire, and assumes known initial conditions with no modeling uncertainties and no external inputs present. It does however lay down the abstract framework for the study of a wide class of infinite dimensional systems with unbounded state and input operators. Such a class of systems includes the Pritchard-Salamon class [19,39] of infinite dimensional systems.

Future direction would involve failures in actuators and in the form of nonlinear dynamics (as opposed to the current case of linear perturbations with linearly parametrized operators) or exogenous failures. This could possibly employ neural networks as used in the finite dimensional case in [51]. As it is often the case, restricted plant information is available, which means that only a noise-corrupted system output is available to assess failures in the system. This type of failure would be studied in the context of flexible structures. Some attempts 


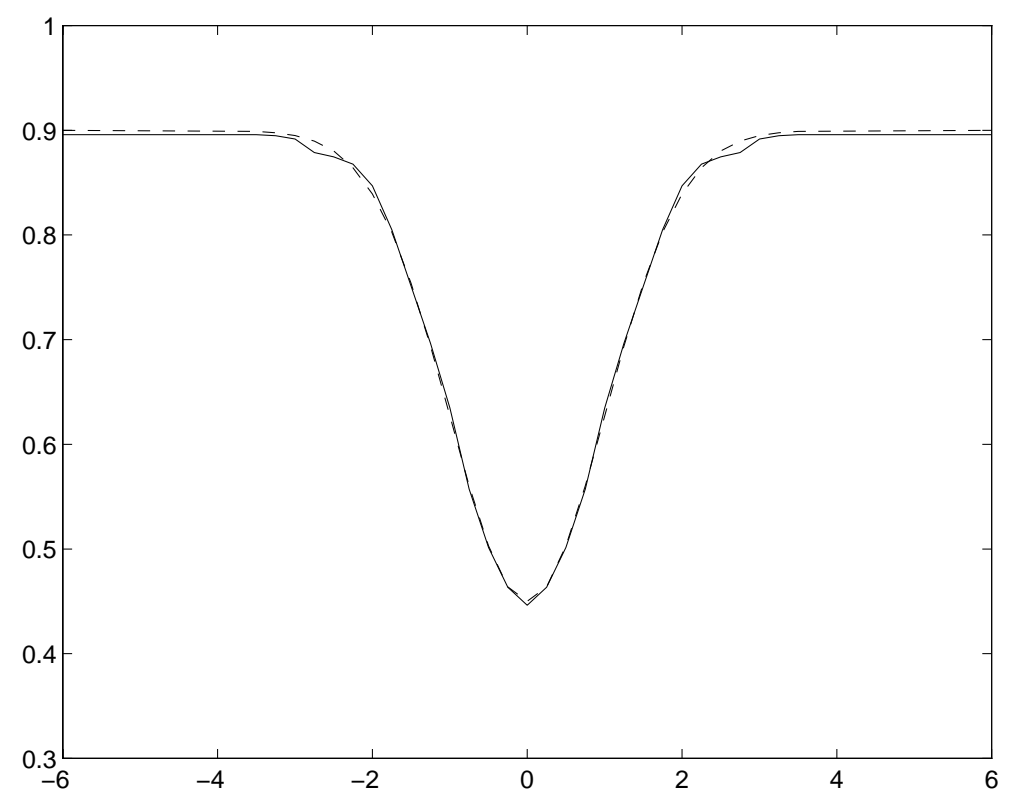

Figure 11. Example 4; Parameter $\theta(r)$ (dashed) and its adaptive estimate $\widehat{\theta}(r)$ (solid) at $t=30 \mathrm{sec}$.

were made to detect the failure in a model for a nonlinear beam in [23-25] and in [22] to detect failures in thermal processes.

Another avenue of interest involves the detection of actuator failure and consequent controller design for the accommodation of flexible structures. Some preliminary studies on this appeared in [1,2,26-29]. Many flexible structures are using smart actuators and sensors for control and observation. These sensors and actuators need to be monitored in order to detect their failures. On-line schemes for actuator/sensor failure detection are therefore needed to accommodate these intelligent structures. Some of the difficulties for these partially observable nonlinear systems lie in the existence and uniqueness of solutions [2] and the strict identifiability conditions that must be imposed in order to avoid false alarms.

The author would like to gratefully thank Professor I.G. Rosen of the University of Southern California for valuable discussions regarding the coding of some of the examples and Professor M.M. Polycarpou of the University of Cinncinnati for fruitful discussions regarding model-based diagnosis schemes using neural networks. Furthermore the author would like to thank Professor Constantine Dafermos of Brown University, Professor Kazufumi Ito of North Carolina State University, Professor Simeon Reich of Technion-Israel Institute of Technology and Professor Ruth Curtain of University of Groningen to issues pertaining to well-posedness of the on-line estimator. Lastly, the author would like to thank Professor C.D. Charalambous of McGill University for valuable input regarding an alternative view of failure time profiles with a stochastic flavor.

\section{REFERENCES}

[1] A. Ackleh, M.A. Demetriou and S. Reich, Detection and accommodation of second order distributed parameter systems with abrupt changes in input term: Existence and approximation, in Proc. of the 6th IEEE Mediterranean Conference on Control and Systems. Alghero, Sardinia (1998).

[2] A.S. Ackleh, S. Aizicovici, M.A. Demetriou and S. Reich, Existence and uniqueness of solutions to a second order nonlinear nonlocal hyperbolic equation, in Proc. of International Workshop on Differential Equations and Optimal Control. Marcel Dekker (2001).

[3] R.A. Adams, Sobolev Spaces. Academic Press, New York (1975). 
[4] N.U. Ahmed and K.L. Teo, Optimal Control of Distributed Parameter Systems. North Holland, New York (1981).

[5] A.M. Annaswamy and K.S. Narendra, Adaptive control of a first order plant with time-varying parameter, in Proc. of the 1989 American Control Conference (1989) 975-980.

[6] - Adaptive control of simple time-varying systems, in Proc. of the 28th IEEE Conference on Decision and Control. Tampa, Florida (1989) 1014-1018.

[7] O. Axelsson and V.A. Barker, Finite Element Solutions of Boundary Value Problems. Academic Press, Orlando, Florida (1984).

[8] M.J. Balas, Finite dimensional direct adaptive control for discrete-time infinite dimensional linear systems, in Proc. of the 33rd IEEE Conference on Decision and Control. Lake Buena Vista, FL, USA (1994) 3424-3429.

[9] H.T. Banks and M.A. Demetriou, Adaptive parameter estimation of hyperbolic distributed parameter systems: Non-symmetric damping and slowly time varying systems. ESAIM: COCV 3 (1998) 133-162.

[10] H.T. Banks and K. Kunisch, Estimation Techniques for Distributed Parameter Systems. Birkhäuser, Boston (1989).

[11] H.T. Banks, R.C. Smith and Y. Wang, Smart Material Structures: Modeling, Estimation and Control. Wiley, Masson, Paris (1996).

[12] M. Basseville and I. Nikiforov, Detection of abrupt changes: Theory and applications. Prentice Hall, Englewood Cliffs, New Jersey (1993).

[13] J. Baumeister, W. Scondo, M.A. Demetriou and I.G. Rosen, On-line parameter estimation for infinite dimensional dynamical systems. SIAM J. Control Optim. 35 (1997) 678-713.

[14] A. Bensoussan, G. Da Prato, M.C. Delfour and S.K. Mitter, Representation and Control of Infinite Dimensional Systems, Vols. I, II. Birkauser, Boston-Basel-Berlin (1992).

[15] M. Böhm, M.A. Demetriou, I.G. Rosen and S. Reich, Model reference adaptive control of distributed parameter systems. SIAM J. Control Optim. 36 (1998).

[16] S.C. Brenner and L.R. Scott, The Mathematical Theory of Finite Element Methods. Springer-Verlag, New York (1994).

[17] C.D. Charalambous and J. Hibey, Conditional densities for continous-time nonlinear hybrid systems with applications to fault detection. IEEE Trans. Automat. Control 44 (1999) 2164-2169.

[18] R.F. Curtain and J.C. Oostveen, Riccati equations for strongly stable, bounded linear systems. Automatica 34 (1998) $953-967$.

[19] R.F. Curtain and H.J. Zwart, An Introduction to Infinite Dimensional Linear Systems Theory. Springer-Verlag, Berlin, Texts Appl. Math. 21 (1995).

[20] M.A. Demetriou, Adaptive Parameter Estimation of Abstract Parabolic and Hyperbolic Distributed Parameter Systems, Ph.D. Thesis, Department of Electrical Engineering - Systems. University of Southern California, Los Angeles, California (1993).

[21] — Model reference adaptive control of slowly time-varying parabolic distributed parameter systems, in Proc. of the 33rd Conference on Decision and Control. Lake Buena Vista, Florida (1994).

$[22] —$ - Fault diagnosis for a parabolic distributed parameter system, in Proc. of the 13th World Congress. International Federation of Automatic Control, San Francisco, California, July (1996).

[23] M.A. Demetriou and B.G. Fitzpatrick, On line estimation of stiffness in nonlinear beam models with piezoceramic actuators, in Proc. of the 1995 ASME Fifteenth Biennial Conference on Mechanical Vibration and Noise \& 1995 ASME Design Technical Conferences. Boston, Mass (1995).

[24] — Results on the adaptive estimation of stiffness in nonlinear beam models, in Proc. of the 3rd IEEE Mediterranean Symposium on New Directions in Control and Automation. Limassol, CYPRUS (1995).

[25] — An adaptive change detection scheme for a nonlinear beam model. Kybernetika 33 (1997) 103-120.

[26] M.A. Demetriou and M.M. Polycarpou, Fault accommodation of output-induced actuator failures for a flexible beam with collocated input and output, in Proc. of the 5th IEEE Mediterranean Conference on Control and Systems. Paphos, CYPRUS (1997).

[27] - Fault detection and diagnosis of a class of actuator failures via on-line approximators, in Proc. of the 1997 36th IEEE Conference on Decision and Control. San Diego, CA (1997).

[28] — Fault diagnosis of output-induced actuator failures for a flexible beam with collocated input and output, in Proc. of the IFAC Symposium on Fault Detection, Supervision and Safety for Processes (SAFEPROCESS). Hull, England (1997).

[29] - Fault detection, diagnosis and accommodation of dynamical systems with actuator failures via on-line approximators, in Proc. of the 1998 American Control Conference. Philadelphia, PA (1998).

[30] - Incipient fault diagnosis of dynamical systems using on-line approximators. IEEE Trans. Automat. Control 43 (1998) $1612-1617$

[31] M.A. Demetriou and I.G. Rosen, On the persistence of excitation in the adaptive identification of distributed parameter systems. IEEE Trans. Automat. Control 39 (1994) 1117-1123.

[32] — Robust adaptive estimation schemes for parabolic distributed parameter systems, in Proc. of the 36th Conference on Decision and Control. San Diego, California, USA (1997) 3448-3453.

[33] P.M. Frank, Fault diagnosis in dynamic systems using analytical and knowledge-based redundancy - a survey and some new results. Automatica 26 (1990) 459-474.

[34] J.J. Gertler, Survey of model-based failure detection and isolation in complex plants. IEEE Control System Magazine 8 (1988) $3-11$. 
[35] J.J. Gertler, Fault Detection and Diagnosis in Engineering Systems. Marcel Dekker, New York (1998).

[36] M. Green and D.J. Limebeer, Linear Robust Control. Prentice Hall, Englewood Cliffs, New Jersey (1995).

[37] P.A. Ioannou and J. Sun, Robust Adaptive Control. Prentice Hall, Englewood Cliffs, NJ (1995).

[38] R. Isermann, Process fault detection based on modeling and estimation methods: A survey. Automatica 20 (1984) $387-404$.

[39] B.V. Keulen, $H_{\infty}$-Control for Distributed Parameter Systems: A State-Space Approach. Birkhäuser, Boston-Basel-Berlin (1993).

[40] T. Kobayashi, Global adaptive stabilization of infinite-dimensional systems. Systems Control Lett. 9 (1987) $215-223$.

[41] - Finite dimensional adaptive control for infinite dimensional systems. Internat. J. Control 48 (1988) $289-302$.

[42] - Input-output representations of spectral systems and adaptive controls. Internat. J. Systems Sci. 19 (1988) $713-732$.

[43] M. Krstic, I. Kanellakopoulos and P. Kokotovic, Nonlinear and adaptive control design. Wiley, New York (1995).

[44] J.L. Lions, Optimal Control of Systems Governed by Partial Differential Equations. Springer-Verlag, New York (1971).

[45] J.L. Lions and E. Magenes, Non-Homogeneous Boundary Value Problems, I. Springer-Verlag, New York (1972).

[46] G.P. Liu and R.J. Patton, Eigenstructure Assignment for Control System Design. John Wiley \& Sons, Chichester (1998).

[47] D.G. Luenberger, An introduction to observers. IEEE Trans. Automat. Control 16 (1971) 596-602.

[48] K.S. Narendra and A.M. Annaswamy, Stable Adaptive Systems. Prentice Hall, Englewood Cliffs, NJ (1989).

[49] R.J. Patton, Robust model-based fault diagnosis: The state of the art, in Proc. of the IFAC Symposium on Fault Detection, Supervision and Safety for Processes (SAFEPROCESS). Espoo, Finland (1994) 1-24.

[50] A. Pazy, Semigroups of Linear Operators and Applications to Partial Differential Equations. Springer-Verlag, New York (1983).

[51] M.M. Polycarpou and A.J. Helmicki, Automated fault detection and accomodation: A learning systems approach. IEEE Trans. Systems Man Cybernet. 25 (1995) 1447-1458.

[52] M.M. Polycarpou and P.A. Ioannou, Neural networks as on-line approximators of nonlinear systems, in Proc. of the 31st IEEE Conference on Decision and Control. San Antonio, Texas (1993) 7-12.

[53] - Stable nonlinear system identification using neural network models, in Neural Networks in Robotics, edited by G. Bekey and K. Goldberg. Kluwer Academic Publishers (1993) 147-164.

[54] M.M. Polycarpou and A.T. Vemuri, Learning methodology for failure detection and accomodation. Control Systems Magazine, special issue on Intelligent Learning Control 15 (1995) 16-24.

[55] V.M. Popov, Hyperstability of Control Systems. Springer-Verlag, Berlin (1973).

[56] L. Praly, G. Bastin, J.B. Pomet and Z.P. Jiang, Adaptive stabilization of nonlinear systems, in Foundations of Adaptive Control, edited by P.V. Kokotovic. Springer-Verlag (1991) 347-433.

[57] M.H. Schultz, Spline Analysis. Prentice-Hall, Englewood Cliffs, NJ (1973).

[58] L.F. Shampine, Numerical Solution of Ordinary Differential Equations. Chapman \& Hall, New York (1994).

[59] R.E. Showalter, Hilbert Space Methods for Partial Differential Equations. Pitman, London (1977).

[60] H. Tanabe, Equations of Evolution. Pitman, London (1979).

[61] S. Townley, Simple adaptive stabilization of output feedback stabilizable distributed parameter systems. Dynam. Control $\mathbf{5}$ (1995) 107-123.

[62] K.S. Tsakalis and P.A. Ioannou, Time-Varying Systems: Control and Adaptation. Prentice Hall, Englewood Cliff, NJ (1993).

[63] J. Wen and M. Balas, Robust adaptive control in Hilbert space. J. Math. Anal. Appl. 143 (1989) 1-26.

[64] J. Wloka, Partial Differential Equations. Cambridge University Press, Cambridge (1987). 